\title{
Convection due to an unstable density difference across a permeable membrane
}

\author{
BABURAJ A. PUTHENVEETTIL ${ }^{1}$ \\ AND JAYWANT H. ARAKERI ${ }^{2}$ \\ ${ }^{1}$ Department of Applied Mechanics, Indian Institute of Technology Madras, Chennai, India \\ ${ }^{2}$ Department of Mechanical Engineering, Indian Institute of Science, Bangalore, India \\ jaywant@mecheng.iisc.ernet.in
}

(Received 30 April 2007 and in revised form 7 May 2008)

We study natural convection driven by unstable concentration differences of sodium chloride $(\mathrm{NaCl})$ across a horizontal permeable membrane at Rayleigh numbers $(\mathrm{Ra})$ of $10^{10}$ to $10^{11}$ and Schmidt number $(S c)=600$. A layer of brine lies over a layer of distilled water, separated by the membrane, in square-cross-section tanks. The membrane is permeable enough to allow a small flow across it at higher driving potentials. Based on the predominant mode of transport across the membrane, three regimes of convection, namely an advection regime, a diffusion regime and a combined regime, are identified. The near-membrane flow in all the regimes consists of sheet plumes formed from the unstable layers of fluid near the membrane. In the advection regime observed at higher concentration differences $(\Delta C)$ across the membrane, there is a slow overturning through-flow across the membrane; the transport across the membrane occurs mostly by advection. This phenomenology explains the observed $N u_{b} \sim R a^{2} / S c$ scaling of the Nusselt number. The planforms of sheet plumes near the membrane show a dendritic structure due to the combined influence of the mean shear due to the large-scale flow and the entrainment flow of the adjacent plumes. The near-membrane dynamics show initiation, elongation and merger of plumes; a movie is available with the online version of the paper. Increase in $R a$ results in a larger number of closely and regularly spaced sheet plumes. The mean plume spacing in the advection regime $\bar{\lambda}_{b}$, is larger than the mean plume spacing in Rayleigh-Bénard convection $(\bar{\lambda})$, and shows a different $R a$-dependence. The plume spacings in the advection regime $\left(\lambda_{b}\right)$ show a common log-normal probability density function at all $R a$. We propose a phenomenology which predicts $\bar{\lambda}_{b} \sim \sqrt{Z_{w} Z_{V_{i}}}$, where $Z_{w}$ and $Z_{V_{i}}$ are, respectively, the near-wall length scales in Rayleigh-Bénard convection (RBC) and due to the advection velocity. In the combined regime, which occurs at intermediate values of $\Delta C$, the flux scales as $(\Delta C / 2)^{4 / 3}$. At lower driving potentials, in the diffusion regime, the flux scaling is similar to that in turbulent RBC.

\section{Introduction}

In turbulent natural convection over horizontal surfaces, small changes to the near-wall phenomena can have drastic effects. A weak shear is known to change the flux scaling whereas rough surfaces increase the flux substantially. The present work investigates some effects of another modification of the near-wall phenomena in high-Rayleigh-number $(R a)$ turbulent free-convection, namely, a weak normal flow through the horizontal walls. The present study could also be looked upon as a 
Rayleigh-Taylor instability where the mixing is considerably slowed down by a permeable membrane.

Unresolved issues remain for the flux scaling, the nature of buoyancy-generated turbulence, and the near-wall dynamics in high $R a$ convection. Many of these issues are discussed in detail in Adrian, Ferreira \& Boberg (1986), Siggia (1994), Kadanoff (2001) and Puthenveettil \& Arakeri (2005). The dimensionless parameters that characterize turbulent natural convection are the Rayleigh number, $R a=g \beta_{T} \Delta T H^{3}$ / $(v \alpha)$, the Prandtl number, $\operatorname{Pr}=v / \alpha$, and the aspect ratio, $A R=L / H$. Here, $g$ is the acceleration due to gravity, $\beta_{T}$ is the coefficient of thermal expansion, $\Delta T$ is the temperature difference between the walls, $H$ is the fluid layer height, $v$ is the kinematic viscosity, $\alpha$ is the thermal diffusivity and $L$ is the horizontal dimension of the fluid layer. In the case of unsteady Rayleigh-Bénard convection (RBC), the ratio of top to bottom fluxes is an additional parameter. The dimensionless flux given by the Nusselt number, $N u=q_{T} /(\alpha \Delta T / H)$, depends on the above parameters, where $q_{T}$ is the kinematic heat flux, $Q / \rho C_{p}, Q=-k_{f}(\partial T / \partial Y)_{Y=0}$, the heat flux, $\rho$ is the fluid density, $C_{p}$ is the specific heat at constant pressure, $k_{f}$ is the thermal conductivity of the fluid, $T$ is the temperature of the fluid and $Y$ is the vertical coordinate direction. If a species is used to create the density difference, then the species diffusivity $D$ replaces $\alpha$, the coefficient of salinity $\beta$ replaces $\beta_{T}$, the Schmidt number $(S c=v / D)$ replaces $P r$, the concentration difference between the walls replaces $\Delta T$, and the mass flux $q$ is equivalent to $q_{T}$.

It is well known that the near-wall region plays a major role in deciding the flux scaling in turbulent natural convection at high $R a$. The experimentally observed scaling law is $N u \sim R a^{n}$ where $n$ shows a large variation between 0.20 to 0.382 , with the majority of the exponents being slightly less than 0.3 (Siggia 1994; Niemela et al. 2000; Chavanne et al. 2001; Xia, Lam \& Zhou 2002; Sun et al. 2005; Niemela \& Sreenivasan 2006). The exponent being close to $1 / 3$ implies that the flux is predominantly determined by the near-wall phenomena, but not completely so. The anomalous flux scaling $(n \neq 1 / 3)$ is expected to be due to the modification of the near-wall phenomena. At large $R a$, this modification is thought to be due to the nearwall shear created by a large-scale flow generated by the system. Modification of the near-wall boundary layers by other means such as roughness elements is also seen to increase the flux substantially (Du \& Tong 2000; Stringano, Pascazio \& Verzicco 2006). Another way the near-wall boundary layers can be modified is by the presence of a wall normal flow; we study this phenomenon, for the first time, in the present paper.

We study turbulent natural convection caused by a denser fluid layer (brine) lying over a lighter fluid layer (distilled water); the two fluids in square-cross-section tanks are separated by a horizontal micro-porous membrane. The gravitational potential across the membrane owing to the presence of brine above water drives the flow, which is resisted by the micro-porous membrane. High $R a\left(\sim 10^{11}\right)$ and $S c(\sim 600)$ are achieved owing to the low molecular diffusivity of sodium chloride $(\mathrm{NaCl})$. There are two limiting cases for the present study. In the absence of a membrane, we obtain the familiar Rayleigh-Taylor instability (see e.g. Young et al. 2001) followed by eventual overturning and mixing of the two fluids. The other limit is a membrane with small pore sizes where the transport across the membrane is merely by diffusion. In this case, the transport above and below the membrane becomes similar to turbulent natural convection above flat horizontal surfaces; these details are discussed in Puthenveettil \& Arakeri (2005, hereinafter referred to as PA). The present study looks at the middle range between these two limits. At sufficiently large pore sizes in the membrane ( $35 \mu \mathrm{m} \times 35 \mu \mathrm{m}$ in the present case) and for a range of concentration differences, the 
transport across the membrane becomes dominated by advection, i.e. by a flow across the membrane. The advection across the membrane is not imposed externally, but generated by the unstable system itself. There is a continuous overturning of the top and bottom fluids, very slow compared to that obtained in Rayleigh-Taylor instability experiments. In this case, the transport from the membrane is not purely by diffusion as it is from the wall in turbulent $\mathrm{RBC}$, but has an additional advective component due to the flow through the membrane. Note that the advection velocity through the membrane is very small $\left(\sim 0.002 \mathrm{~cm} \mathrm{~s}^{-1}\right)$ compared to the buoyancyinduced convection velocity in the bulk $\left(\sim 0.5 \mathrm{~cm} \mathrm{~s}^{-1}\right)$. Hence, similar to turbulent $\mathrm{RBC}$, unstable liquid layers are formed near the membrane surface. However, the nature of these near-membrane boundary layers and the scaling of flux are changed owing to the advection across the membrane. In this paper, we focus on the effect of this advection on the flux scaling and the near-wall structures.

There are few previous studies on related phenomena, none on the same phenomenon. The effect of wall normal flow on the stability of a fluid layer confined between a hot bottom and a cold top plate has been investigated by Shvartsblat (1969) and Nield (1987). Boundary-layer effects are not considered in these studies. Gill, Zeh \& Casal (1965), Clarke \& Riley (1975) and Afzal \& Hussain (1986) obtained similarity solutions of natural convection boundary layers on a horizontal surface with a wall-normal advective flow. However, a restrictive dependence of the wall normal advection velocity on the distance from the leading edge has to be assumed in order to obtain similarity solutions. Two-dimensional solutal convection in a Hele-Shaw cell, with external pumping at the boundaries, was investigated by Bizon et al. (1997) to obtain convection patterns and the mean concentration profile. These studies are not directly relevant to the present study as we concentrate on a spatially uniform advection velocity through the membrane in a wide square-cross-section tank with no external pumping at very high $R a$.

The paper is organized as follows. We describe the experimental set-up in $\S 2.1$ and the techniques for the visualization of the structures near the membrane in $\S 2.2$. The data analysis for calculating the flux and the justifications for the quasi-steady assumption are described in $\S 2.3$ and $\S 2.4$ respectively. In $\S 3$, we introduce the expected driving potentials and the possible types of flow which give rise to these concentration differences. The observations of flux scaling in $\S 4$ help us to infer three regimes of convection, namely, the advection regime, the diffusion regime and a combined regime. We then focus on the advection regime in $\S \S 5,6$ and 7 . The plume structure and the convection patterns are described in $\S 5.1$, the plume dynamics near the membrane in $\S 5.2$ and the phenomenology for the cubic scaling of flux in $\S 5.3$. In $\S 6$, we analyse the mean and the statistics of plume spacings. A model for the mean plume spacings in the advection regime is described in $\S 7$.

\section{Experimental set-up and data analysis}

\subsection{Set-up}

A schematic of the experimental set-up is shown in figure 1(a). The set-up consists of two glass compartments of square cross-section, arranged one on top of the other with a fine stretched membrane fixed horizontally in between them. We use a $140 \mathrm{~S}$ Swedish Nylobolt screen-printing mesh made of Nylon wires of $35 \mu \mathrm{m}$ diameter, having square pores of side $35 \mu \mathrm{m}$. Figure $1(d)$ shows the $200 \times$ scanning electron microscope $(\mathrm{SEM})$ image of the membrane surface. The open area factor of the membrane, $\Gamma=0.25$, and its thickness is $l_{m}=70 \mu \mathrm{m}$. In the experiment, the bottom tank is first filled with 
(a)

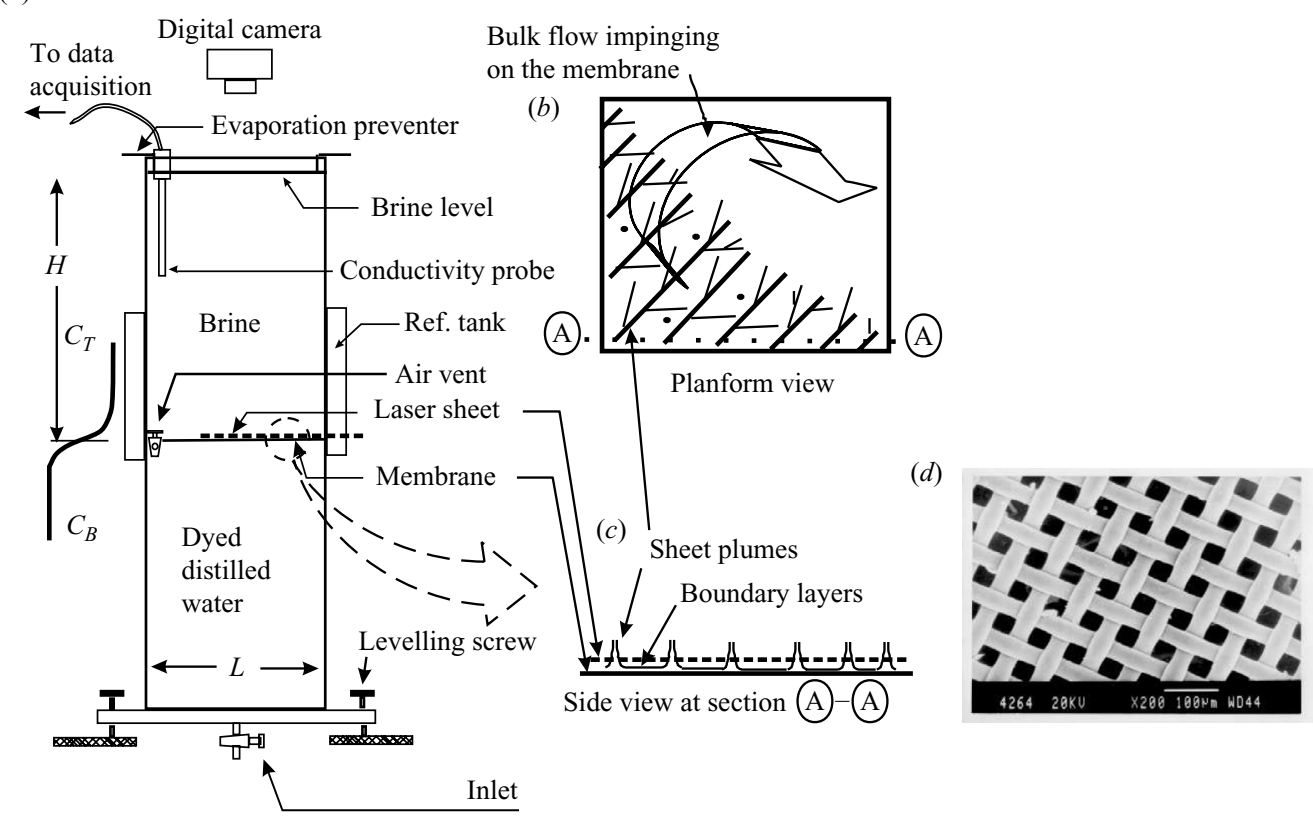

Figure 1. (a) The experimental set-up. (b) The planform view of the near-membrane plume structure as seen at the intersection of a horizontal laser sheet. (c) The side view in a vertical plane showing rising sheet plumes and their intersection with the horizontal laser sheet. $(d)$ SEM image $(200 \times)$ of the 140 S Swedish Nylobolt membrane.

distilled water tagged with a small amount of sodium fluoresceine; the top tank is then filled with brine. A thin walled Perspex tank, with a thin glass plate on top of its sponge bottom, and just fitting into the top compartment, is kept within the top compartment during the filling process. The glass plate and sponge prevent mixing due to direct impingement of brine on the membrane while filling the top tank. After filling the top tank up to the height of the bottom tank, this Perspex tank is removed slowly to initiate the experiment. A thin transparent Perspex sheet of the dimensions of the test-section cross-section is kept floating on the brine solution to prevent evaporation and to produce similar boundary conditions in the two compartments. The whole assembly is mounted on a levelling table to make the membrane horizontal. Transport of salt across the membrane sets up unstable layers on either side of the membrane and causes convection in the two tanks. No external circulation is imposed in either of the tanks; it is a run down experiment. Convection eventually stops after density equalization in the tanks, which typically takes about 2 days. Experiments are conducted with a $23 \mathrm{~cm}$ high layer of top and bottom tank solution in a tank of $15 \mathrm{~cm} \times 15 \mathrm{~cm}$ cross-section $(A R=0.65)$, with starting top-tank concentrations of $10 \mathrm{~g}^{-1}, 7 \mathrm{~g}^{-1}$ and $3 \mathrm{~g}^{-1}$ to study the plume structure at different $R a$. Further details of the experimental set-up and procedure are discussed in Puthenveettil (2004).

\subsection{Diagnostics}

A horizontal laser sheet, expanded and collimated from a $5 \mathrm{~W}$ Spectra Physics Ar-Ion laser Stabilite ${ }^{\mathrm{TM}} 2017$ is passed just above $(<1 \mathrm{~mm})$ the membrane to visualize the planform structures near the memebrane. The dye in the bottom-tank solution, while convecting upward, fluoresces on incidence of the laser light sheet to make the plume 

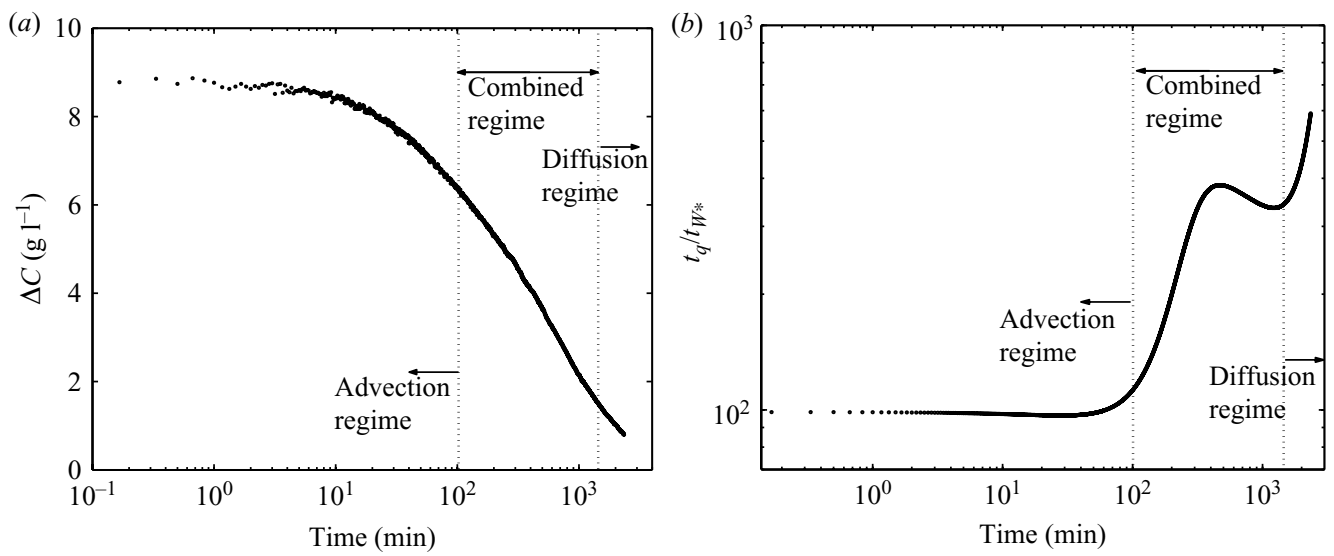

FiguRE 2. (a) Variation of the driving potential $\Delta C$ (2.1) in a typical experiment. (b) Variation of the ratio of the time scale for the change of flux $t_{q}(2.5)$, to the time scale of one large-scale circulation $t_{W_{*}}=H / W_{*}$ in a typical experiment.

structure visible (figure 1c). The diffusion coefficient of salt is about three times the diffusion coefficient of the dye $(S c=600$ for salt (Lide 2001) as against $S c=2000$ (Buch \& Dahm 1996) for the dye). The density change due to the addition of the dye is negligible owing to the very low concentration (1.2 p.p.m.) used. Hence, the dye can be considered as a passive tracer in the fluid, carried around by the motion induced by the density changes due to the salt concentration variations. Sodium fluoresceine has an absorption spectra peaking at $490 \mathrm{~nm}$ and an emission spectra peaking at $520 \mathrm{~nm}$ (Crimaldi 1997). We use the $488 \mathrm{~nm}$ wavelength of the laser to maximize the absorption of light by the dye. A visible long-pass filter glass, Coherent optics OG-515, is used to block any scattered laser light and allow the emitted fluorescence to pass through. The images are captured on a digital handycam Sony DCR PC9E.

\subsection{Measurement of concentration and flux}

We measure the concentration of salt in the top tank with time. From this measurement, we obtain both the flux of salt and the concentration difference across the membrane. We assume that the fluids in both the compartments are well mixed in the region away from the membrane, and hence can be represented by the top-tank concentration $C_{T}(t)$ and the bottom-tank concentration $C_{B}(t)$ at any instant of time $t$. This assumption is generally valid for high $R a$ convection which have thin boundary layers and a well-mixed bulk. We also checked the validity of this assumption from the concentration maps obtained using planar laser-induced fluorescence technique (Dahm \& Dimotakis 1987), see Puthenveettil (2004) for details.

Using mass balance, the concentration difference between the compartments at time $t, \Delta C(t)=C_{T}(t)-C_{B}(t)$ is,

$$
\Delta C(t)=\left(1+\frac{V_{T}}{V_{B}}\right) C_{T}(t)-C_{T}^{0} \frac{V_{T}}{V_{B}},
$$

where $V_{T}$ is the top-tank solution volume, $V_{B}$ is the bottom-tank solution volume and the superscript zero indicates the initial value. The typical variation of the driving potential $\Delta C$ in an experiment is shown in figure $2(a)$. In the present study, the top surface of the fluid layer above the membrane is adiabatic and hence we define

$$
R a_{w}=\frac{g \beta \Delta C_{w} H^{3}}{\nu \alpha}
$$


based on $\Delta C_{w}$, the difference between concentration at the surface of the membrane and in the bulk of the top fluid layer, as the relevant Rayleigh number. As we shall discuss below, $\Delta C_{w}=C_{T}-C_{B}$ in the advection regime; in the diffusive and the combined regimes $\Delta C_{w} \leqslant\left(C_{T}-C_{B}\right) / 2$.

The rate of change of the well-mixed top-tank concentration gives the area averaged net flux of sodium chloride $(\mathrm{NaCl})$ out of the top tank per unit area of the tank cross-section at any instant as,

$$
q(t)=-H \frac{\mathrm{d} C_{T}}{\mathrm{~d} t},
$$

where $H$ is the top (and the bottom) liquid-layer height. Hence, we make transient measurements of the changing well-mixed concentration in the top tank $C_{T}$, to estimate $\Delta C$ and flux from (2.1) and (2.3).

The concentration of $\mathrm{NaCl}$ in the top tank is estimated from the measurement of electrolytic conductivity by ORION SENSORLINK ${ }^{\mathrm{TM}}$ PCM100 conductivity measurement system (ORION 1999). The cell has an outer diameter of $12 \mathrm{~mm}$ and a $0.5 \%$ full scale accuracy in the present measuring range of 2 to $20 \mathrm{mS} \mathrm{cm}^{-1}$, with automatic temperature compensation. The probe is calibrated for cell constant before each experiment, and the measured conductivities converted to $\mathrm{NaCl}$ concentrations using the standard relation from the CRC handbook (Lide 2001). Before each experiment, known $\mathrm{NaCl}$ concentrations made from prepared samples and dilution samples from the top-tank brine solution are measured and compared.

Direct calculation of $\mathrm{d} C_{T} / \mathrm{d} t$ in (2.3) from the measured $C_{T} v$ s. $t$ curve results in excessive noise owing to differentiation. Hence, an exponential decay fit of the form

$$
C_{T}(t)=y_{0}+A_{1} \mathrm{e}^{-t / b_{1}}+A_{2} \mathrm{e}^{-t / b_{2}}+A_{3} \mathrm{e}^{-t / b_{3}}
$$

through the $C_{T}$ vs. $t$ curve, with $\chi^{2}<10^{-3}$, is used to calculate $\mathrm{d} C_{T} / \mathrm{d} t$. Here, $y_{0}, A_{1}$, $b_{1}, A_{2}, b_{2}, A_{3}, b_{3}$ are fit constants. The derivative obtained from the fit was compared with the derivative obtained from the smoothed data in each experiment; the derivative from the smoothed data was close and distributed around that from the fit. In most experiments, concentration measurements and visualizations are conducted simultaneously.

\subsection{Quasi-steady approximation}

Note that the present study is a case of unsteady non-penetrating convection (UNP) (Adrian et al. 1986) whereas most of the investigations in the literature are conducted for steady RBC. For constant flux, both UNP and RBC are similar with respect to their turbulence characteristics (Adrian et al. 1986; Theerthan \& Arakeri 2000). In the present case, the mean concentration and the flux keep changing throughout the experiment. Even though the flux is not constant, the situation can be considered as quasi-steady by understanding the relevant time scales in the system. The time scale of the decrease of flux is

$$
t_{q}=q / \frac{\mathrm{d} q}{\mathrm{~d} t}=\frac{\mathrm{d} C_{T}}{\mathrm{~d} t} / \frac{\mathrm{d}^{2} C_{T}}{\mathrm{~d} t^{2}},
$$

which varies from $75 \mathrm{~min}$ at the start to $1925 \mathrm{~min}$ at the end of a typical experiment. The time taken for one large-scale circulation, $t_{W_{*}}=H / W_{*}$ varies between $46 \mathrm{~s}$ to $196 \mathrm{~s}$ from the beginning to the end of the experiment. Here,

$$
W_{*}=(g \beta q H)^{1 / 3}
$$


(a)

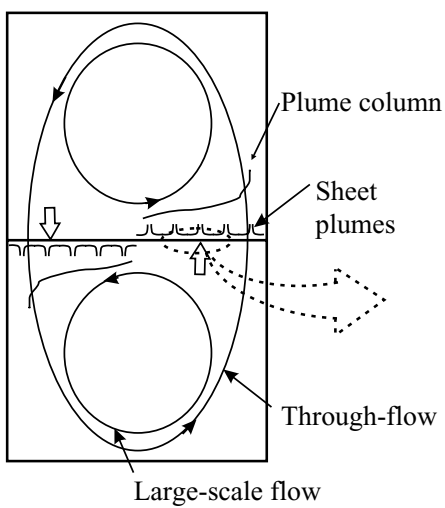

(b)

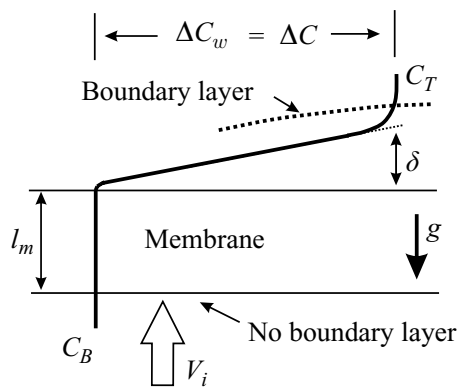

FiguRE 3. (a) Flow in the advection regime. The slow overturning circulatory motion across the membrane between the tanks is termed as through-flow. Through-flow creates advection across the membrane. The bulk flow in the top and bottom tank is termed large-scale flow. The large-scale flow creates the near-membrane shear. Plumes are seen on the opposite side of the through-flow impingement. (b) The concentration profile on the right-hand upper part of the membrane where advection is from bottom to top. The whole concentration difference acts on the opposite side of the through-flow impingement. The transport across the membrane is by advection (indicated by open arrows).

is the characteristic velocity scale in the bulk known as the Deardorff velocity scale (Deardorff 1970) which gives an appropriate estimate of the large-scale flow velocity in high-Rayleigh-number convection (see PA). Figure $2(b)$ shows the variation of the ratio $t_{q} / t_{W_{*}}$ with time in a typical experiment. The smallest value of $t_{q} / t_{W_{*}}$ is about 100 in the advection regime. This means that even when the flux is changing at its fastest rate, the flux is literally constant over many large-scale circulation times. Therefore, many single large-scale circulations sees a constant flux and a constant driving potential. All other time scales in the experiment are much smaller than the time scale for one large-scale circulation; the experiment is hence quasi-steady. A steady experiment, similar to that by Bizon et al. (1997), could also be conducted. However, understanding that the wide separation of time scales makes the experiment quasi-steady, the present simple set-up is felt to be sufficient.

\section{Introduction to the types of flow}

The analysis of our data is based on the understanding that three regimes, differentiated based on the nature of transport across the membrane, can occur in the experiments. The predominant mode of transport across the membrane could be advection, a combination of advection and diffusion, or pure diffusion. Each of these transport modes will engender a different concentration profile across the membrane. The expected concentration profiles in the three regimes, along with the type of flow that create the concentration profile, are shown in figures 3 to 5. We name the regimes as $(a)$ the advection regime, $(b)$ the diffusion regime and $(c)$ the combined regime; the name indicates the dominant mode of transport across the membrane. The inference of these regimes is validated later from our experimental observations.

In the advection regime, there is a slow overturning motion which is up across one half of the membrane surface and down across the other half. Hereinafter, we call this circulation connecting the tanks through the membrane as through-flow. A possible bulk flow that could create such a regime is shown in figure $3(a)$, the corresponding 
(a)

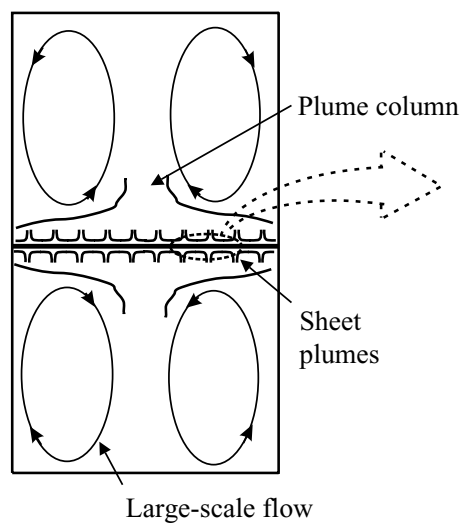

(b)

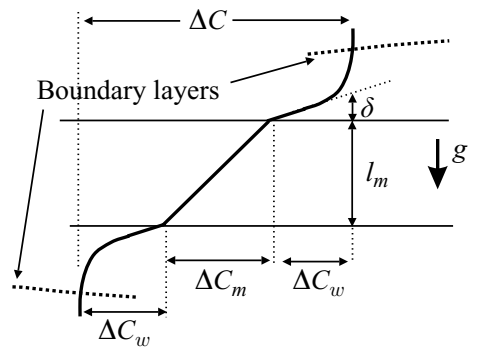

FIgURE 4. (a) Flow in the diffusion regime. Plumes cover the upper and the lower region of the membrane completely. Two large-scale flow cells are shown in each tank. Depending on $R a$ and $A R$, the number of cells could be different (see PA). (b) The concentration profile on the upper and the lower region of the membrane. The transport across the membrane is by diffusion. There is no through-flow.

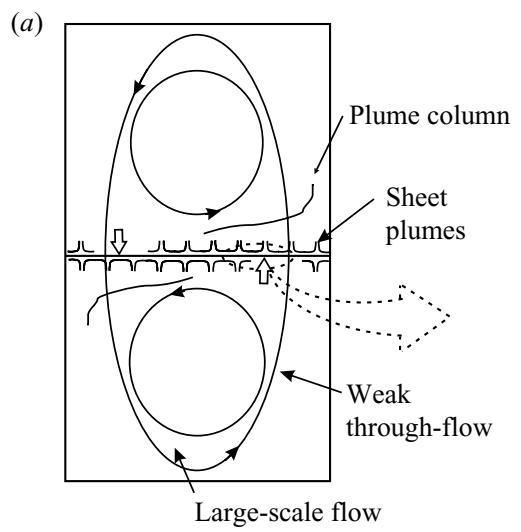

(b)

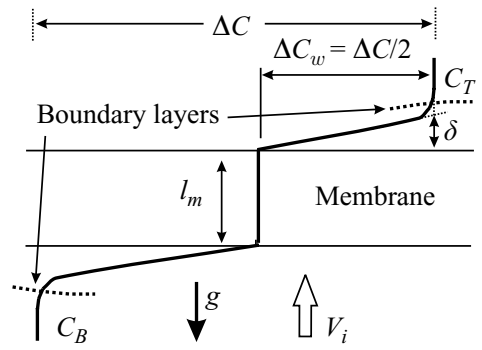

Figure 5. (a) Flow in the combined regime. The through-flow is of lower strength and in smaller patches than that in figure 3(a), creating a smaller area that is free of plumes on the side of impingement. The large-scale flow, which is weaker than that in figure 3(a), creates the near-membrane shear. $(b)$ The effective concentration profile on the upper and the lower region of the membrane.

concentration profile in the half where the flow is upwards is shown in figure $3(b)$. The flow through the membrane pores could prevent formation of boundary layers on one side of the membrane; all the concentration drop is on one side (figure $3 b$ ) and the transport across the membrane is by advection. The values of advection velocities at which all the concentration drop occurs on one side of the membrane are discussed in Appendix D. The very small flow through the membrane pores is hereinafter referred to as advection, indicated by open arrows in the figures. In addition, there is a circulatory motion within each tank, which is hereinafter referred to as the large-scale flow.

In the diffusion regime, which occurs at lower driving potentials, the bulk throughflow is effectively non-existent to drive the concentration difference to one side of the membrane. The schematic of the large-scale flow in such a situation is shown in figure $4(a)$. The transport across the membrane will be purely by diffusion, a 
concentration drop occurs across the membrane as shown in figure $4(b)$. This case is investigated in PA. In the combined regime, at driving potentials intermediate to that in the advection and the diffusion regime, the transport across the membrane is due to advection and diffusion. The schematic of the bulk flow in the combined regime, weaker than that in the advection regime, is shown in figure 5(a); the effective concentration profile is shown in figure $5(b)$.

In all these regimes, $\Delta C_{w}$ is the relevant driving potential for convection in the top and the bottom tanks. However, in each regime, the relation of $\Delta C_{w}$ to the well-mixed concentration difference between the tanks, $\Delta C$, is different. In the advection regime, $\Delta C_{w}=\Delta C$, as all the concentration drop occurs in the boundary layer on the opposite side of the impingement of the through-flow (figure $3 b$ ). In the diffusion regime, if

$$
\Delta C_{m}=q l_{m} /(\Gamma D)
$$

is the concentration drop across the membrane owing to diffusion, the effective concentration difference which drives the convection on one side of the membrane would be

$$
\Delta C_{w}=\frac{1}{2}\left(\Delta C-\Delta C_{m}\right)
$$

(see figure $4 b$ ). The combination of advection and diffusion in the combined regime results in an effective concentration on both sides of the membrane; we later find this effective concentration to be $\Delta C_{w}=\Delta C / 2$ (figure $5 b$ ). In each of these three regimes, we measure only the top-tank concentration, the appropriate $\Delta C_{w}$ are then calculated from $\Delta C$. The Rayleigh number $R a_{w}$ for each regime is calculated using the relevant driving potential $\Delta C_{w}$ by (2.2).

The area averaged flux of salt calculated by (2.3) will have local convective, diffusive or both the components at the membrane surface, depending on the convection regime. In $\mathrm{RBC}$, the surface transport is always merely by diffusion. In the present study, the local instantaneous vertical flux at any horizontal location $X$ on the membrane surface is given by,

$$
q_{l}=v C-D \frac{\partial C}{\partial Y}
$$

where $v$ is the vertical component of the instantaneous velocity and $C$ is the instantaneous concentration at the point. The area average of the flux in (3.3) will give the net flux of salt across the membrane defined in (2.3). It may be noted that the area average of the vertical velocity $v$ on the membrane surface will be zero in the advection regime. The first term in (3.3) will be the dominant contribution to the flux in the advection regime whereas only the second term will contribute in the diffusion regime. In the combined regime, the total flux across the membrane will have non-negligible contributions from both the terms in (3.3).

\section{Flux scaling}

Figure 6 shows the variation of the flux with the dimensionless density difference, $\Delta \rho / \rho=\beta \Delta C$, during typical experiments started with initial top-tank concentrations of $C_{T}^{0}=10 \mathrm{~g} \mathrm{l}^{-1}, 7 \mathrm{~g} \mathrm{l}^{-1}$ and $3 \mathrm{~g}^{-1}$. There is a clear change in the slope of the curve at $\Delta \rho / \rho \approx 0.0045$ for the experiment with $C_{T}^{0}=10 \mathrm{~g}^{-1}$, indicating a change in the regime of convection. The $7 \mathrm{~g}^{-1}$ and the $3 \mathrm{gl}^{-1}$ experiments follow the $10 \mathrm{gl}^{-1}$ experimental curve at the corresponding $\Delta \rho / \rho$. (The deviation of the initial part of the curve for the $7 \mathrm{~g}^{-1}$ experiment in figure $6(a)$ from that of the $10 \mathrm{~g}^{-1}$ experiment at the same $\Delta \rho / \rho$ is expected to be an effect of the initial condition in the $7 \mathrm{gl}^{-1}$ experiment. At this 

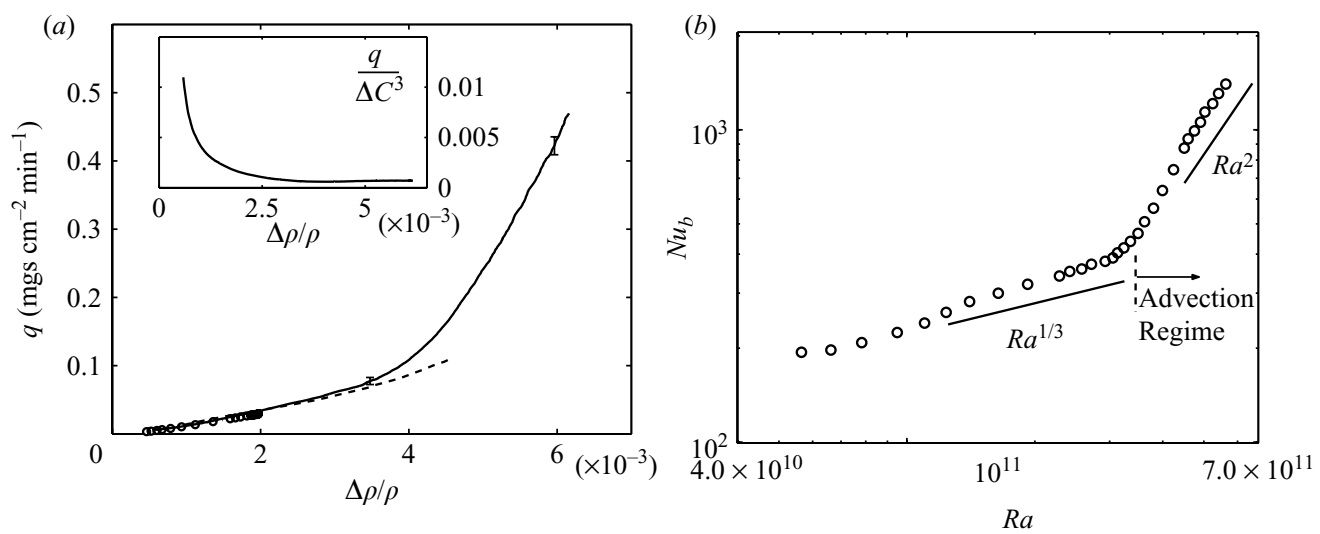

FIGURE 6. (a) Variation of the flux with the dimensionless density difference for experiments with, -,$C_{T}^{0}=10 \mathrm{gl}^{-1} ;--, 7 \mathrm{gl}^{-1} ; \bigcirc, 3 \mathrm{gl}^{-1}$. The inset shows the scaling of flux in the initial part of the $C_{T}^{0}=10 \mathrm{gl}^{-1}$ experiment. $(b)$ Variation of the Nusselt number with the Rayleigh number for a $C_{T}^{0}=10 \mathrm{gl}^{-1}$ experiment.

$\Delta \rho / \rho$, in the $10 \mathrm{gl}^{-1}$ experiment, the bulk flow continues as it was in the advection regime before it slowly changes its nature. However, in the $7 \mathrm{gl}^{-1}$ experiment, the initial $\Delta C$ is not large enough to initiate a bulk through-flow; different regimes seems to be possible at these concentrations depending on the initial conditions.) The inset in figure 6 shows that the flux scales as $\Delta C^{3}$ in the initial part of the $C_{T}^{0}=10 \mathrm{~g} 1^{-1}$ experiment.

Figure $6(b)$ shows the variation of $N u_{b}$ with $R a$, where $N u_{b}=q /(D \Delta C / H)$, is a Nusselt number and $R a=g \beta \Delta C H^{3} /(v \alpha)$ is the Rayleigh number. Three convection regimes can be identified based on the slope of the $N u_{b} v s$. $R a$ curve. In the initial higherconcentration regions, $N u_{b} \sim R a^{2}$ while $N u_{b} \sim R a^{1 / 3}$ for $R a<2 \times 10^{11}$. There is one more change in slope of the curve at $R a \approx 7 \times 10^{10}$.

To explore the presence of the three regimes discussed in $\S 3$, we look at the data of figure $6(a)$, normalized by the near-membrane scales as

$$
R a_{\delta}^{-1 / 3}=\frac{q}{D \Delta C_{w} / Z_{w}}
$$

where, $R a_{\delta}$ is the Rayleigh number based on the diffusion-layer thickness, $\delta=$ $D \Delta C_{w} / q$, and

$$
Z_{w}=\left(\frac{v D}{g \beta \Delta C_{w}}\right)^{1 / 3}
$$

is a near-wall length scale for turbulent natural convection (Theerthan \& Arakeri 2000). As $R a_{\delta}^{-1 / 3}$ is a representation involving only the near-membrane variables, the changes in the near-membrane phenomena will be seen more clearly in terms of $R a_{\delta}^{-1 / 3}$ than in terms of $N u_{b}$. We could expect to identify the three regimes from the variation of $R a_{\delta}^{-1 / 3}$.

$R a_{\delta}^{-1 / 3}$ is a direct measure of the heat flux and varies between only 0.1 and 0.3 for a range of $R a, P r$ and $A R$, and for various types of turbulent natural convection on smooth surfaces (without advection through the walls); the flux is mostly decided by the near-wall phenomena, which remain the same in all these cases (Theerthan \& Arakeri 2000). A correlation $N u=C R a^{n}$ for $\mathrm{RBC}$, rewritten in terms of $R a_{\delta}^{-1 / 3}$ 

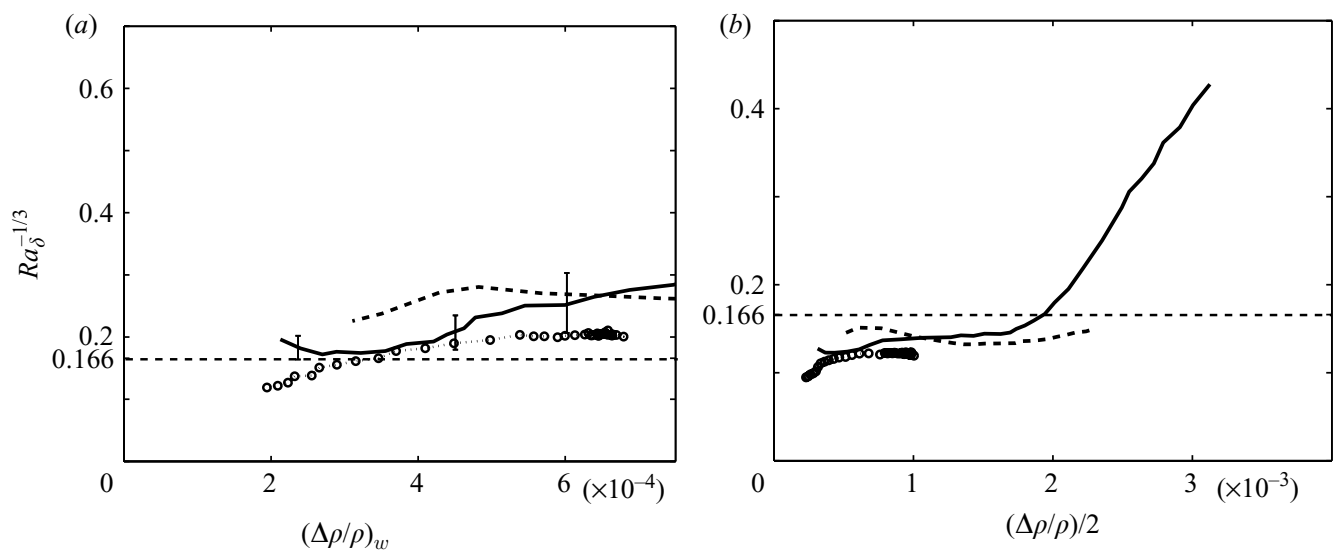

FIGURE 7. Variation of $R a_{\delta}^{-1 / 3}$ with $(\Delta \rho / \rho)_{w}$, the dimensionless density difference between the membrane and the bulk.,$- C_{T}^{0}=10 \mathrm{gl}^{-1} ;---, 7 \mathrm{~g}^{-1} ; \circ, 3 \mathrm{gl}^{-1}$. (a) $\Delta C_{w}=\left(\Delta C-\Delta C_{m}\right) / 2$, i.e. if the concentration profile is as shown in figure $4(b)$. (b) $\Delta C_{w}=\Delta C / 2$, i.e. if the concentration profile is as shown in figure $5(b) . R a_{\delta}^{-1 / 3}$ from the Goldstein et al. (1990) correlation when $q \sim \Delta C_{w}^{4 / 3}$ is 0.166 .

becomes $R a_{\delta}^{-1 / 3}=A R a^{n-1 / 3}$, where $A=2^{4 / 3} C . R a_{\delta}^{-1 / 3}$ is a constant if the flux scales as $\Delta C^{4 / 3}$, i.e. if $N u \sim R a^{1 / 3} \cdot R a_{\delta}^{-1 / 3}=0.166$ for the correlation $N u=0.0659 R a^{1 / 3}$ obtained by Goldstein, Chiang \& See (1990) for $\operatorname{Pr}=2750$ and $10^{8} \leqslant R a \leqslant 10^{12}$. If $n=2 / 7$, then $R a_{\delta}^{-1 / 3}=A R a^{-1 / 21}$, a weak function of $R a$. A similar feature is observed in wallbounded turbulent shear flows where variations of the coefficient of friction $C_{f}$ with Reynolds number are found to be small. In fact, $R a_{\delta}^{-1 / 3}$ is analogous to the $C_{f}$ representation of wall shear stress.

The dependence of the dimensionless flux $R a_{\delta}^{-1 / 3}$ on $(\Delta \rho / \rho)_{w}=\beta \Delta C_{w}$, for experiments started with $C_{T}^{0}=10,7$ and $3 \mathrm{gl}^{-1}$ is shown in figure 7. In figure 7(a), $R a_{\delta}^{-1 / 3}$ is calculated using $\Delta C_{w}=\left(\Delta C-\Delta C_{m}\right) / 2$ whereas it is calculated using $\Delta C_{w}=\Delta C / 2$ in figure $7(b)$. In figure $7(a)$, at driving potentials $(\Delta \rho / \rho)_{w} \leqslant 4 \times 10^{-4}$, the magnitude of $R a_{\delta}^{-1 / 3}$ is approximately constant at around 0.17 , close to the value in RBC; therefore $N u_{b} \sim R a_{w}^{1 / 3}, q \sim \Delta C_{w}^{4 / 3}$ with $\Delta C_{w}=\left(\Delta C-\Delta C_{m}\right) / 2$. In figure $7(b), R a_{\delta}^{-1 / 3}$ is constant at about 0.14 for $0.75 \times 10^{-3} \leqslant(\Delta \rho / \rho) / 2 \leqslant 2 \times 10^{-3}$ for all three experiments, similar to RBC. Therefore, $N u_{b} \sim R a_{w}^{1 / 3}$ and the flux scales as $\left(\Delta C_{w}\right)^{4 / 3}$ with $\Delta C_{w}=\Delta C / 2$ for this range of $\Delta \rho / \rho$.

The behaviour of flux confirms our inference of three regimes of convection.

(a) The flux scales as $\Delta C^{3}$ for $\Delta \rho / \rho \geqslant 4.5 \times 10^{-3}\left(\Delta C \geqslant 6.4 \mathrm{gl}^{-1}\right)$. The scaling is quite different from that in $\mathrm{RBC}$; we expect this difference to be due to advection through the membrane pores. We classify this regime as the advection regime with a concentration profile as shown in figure $3(b)$.

(b) There seems to be a combined regime in the range $1 \times 10^{-3} \leqslant \Delta \rho / \rho \leqslant 3.5 \times 10^{-3}$ $\left(1.5 \mathrm{~g}^{-1} \leqslant \Delta C \leqslant 5 \mathrm{~g}^{-1}\right)$ where $q \sim(\Delta C / 2)^{4 / 3}$ and the magnitude of the flux is the same as $\mathrm{RBC}$, if $\Delta C_{w}=\Delta C / 2$. A concentration profile as shown in figure $5(b)$ would give rise to such a scaling and magnitude of flux.

(c) For $\Delta \rho / \rho<1 \times 10^{-3}\left(\Delta C<1.5 \mathrm{~g}^{-1}\right)$, flux scales as $\Delta C_{w}^{4 / 3}$ similar to that in $\mathrm{RBC}$. The magnitude of the flux for a given $\Delta C$, corrected for the drop across the 
membrane, is also the same as that in $\mathrm{RBC}$ for the same near-wall concentration differences. Hence, we expect the concentration profile in this range of $\Delta C$ to be as shown in figure $4(b)$ and refer to this regime as the diffusion regime.

In experiments started with $C_{T}^{0}=10 \mathrm{gl}^{-1}$, the advection regime occurs first at higher concentrations. $\Delta C$ decreases with time during the experiment; the combined regime appears when $\Delta C$ falls between 5 and $1.5 \mathrm{~g}^{-1}$. Towards the end of an experiment started with $C_{T}^{0}=10 \mathrm{~g}^{-1}$, we observe the diffusion regime at $\Delta C$ lower than $1.5 \mathrm{~g} \mathrm{l}^{-1}$. In the $C_{T}^{0}=7$ and $3 \mathrm{~g}^{-1}$ experiments, as is seen from figure $6(a)$, the combined regime occurs first, followed by the diffusion regime.

Note that in the combined and the diffusion regimes, since the flux normalized by the near-membrane scales (equation (4.1)) remains constant (figure 7), the flux and the near-membrane dynamics are completely determined by the near-membrane diffusion scales. In contrast, as the normalized flux in the advection regime do not remain constant in figure $7(b)$, the flux is expected to be a function of the bulk scales, the membrane properties and the near-membrane scales. The transition point between these regimes is dependent on the membrane properties. The diffusion regime was observed by PA in the range $0.5 \times 10^{11}<R a_{w}<2 \times 10^{11}$ using a membrane with a much smaller pore size $(0.45 \mu \mathrm{m})$ but a larger open area factor $(\Gamma=0.6)$; with the present membrane, we observe a combined regime in this $R a_{w}$ range (see figure 12 below). Thus, it is important to note that the membrane properties, the Rayleigh number and (presumably) the aspect ratio all determine the regime obtained. A larger resistance to through-flow by having a membrane with a smaller pore size will give the diffusion regime for the same driving potential, whereas a smaller resistance will give the advection regime. Even though the total $R a$ range of the experiment is over a decade, the range of $R a$ for each of the regimes is not large; clearly, further studies are required on the range of validity of these flux scalings.

\section{The advection regime}

We have seen that in the initial periods of experiments started with $C_{T}^{0}=10 \mathrm{~g}^{-1}$, the magnitude of flux and its scaling are quite different from that in RBC. We now look at the convection patterns and the near-membrane plume structure in this advection regime and then propose a phenomenology to explain the $\Delta C^{3}$ scaling of flux in this regime of convection.

\subsection{Convection pattern and plume structure}

Figure $8(a)$ shows the commonly observed planform (top view) plume structure near the membrane in the advection regime. The parameters corresponding to the planform are given in table 1 . The figure corresponds to $14.7 \mathrm{~min}$ after the initiation of an experiment with $C_{T}^{0}=10 \mathrm{gl}^{-1}$. The laser sheet is horizontal and just above the membrane. The white lines in the image are the top view of the dyed lighter fluid rising from the membrane surface. The lines represent the bases of the sheet plumes; sheet plumes are continuous sheets of rising (resp. falling) lighter (resp. heavier) columns of fluid. The sheet plumes form and merge. Similar sheet plumes have been observed in RBC at $\mathrm{Pr} \sim 1$ (Zocchi, Moses \& Libchaber 1990; Theerthan \& Arakeri 2000; Funfschilling \& Ahlers 2004; Zhou, Sun \& Xia 2007) and in UNP at high $\operatorname{Pr}$ (PA; Puthenveettil, Ananthakrishna \& Arakeri 2005). Half the membrane area is covered by plumes. Plumes are observed (by shadow graph) on the bottom surface of the membrane corresponding to the plume-free area on the top surface. The plumes become more concentrated in the corner with increasing height. The 
$\Delta C \quad \frac{\Delta \rho}{\rho} \times \quad \Delta C_{w} \quad\left(\frac{\Delta \rho}{\rho}\right)_{w}$ Figure Exp. $\left(\mathrm{gl}^{-1}\right) 10^{-3}\left(\mathrm{gl}^{-1}\right) \times 10^{-3} R a_{w} \times 10^{11}$

Flux

$\left(\mathrm{mg} \mathrm{cm}^{-2} \quad W_{*} \quad V_{i} \quad\right.$ Image size $\left.\mathrm{min}^{-1}\right)\left(\mathrm{cm} \mathrm{s}^{-1}\right) \quad R e \quad\left(\mathrm{~cm} \mathrm{~s}^{-1}\right)(\mathrm{cm} \times \mathrm{cm})$

$\begin{array}{rrlllllllllr}8(a) & 8 \mathrm{j} & 7.4 & 5.2 & 7.4 & 5.2 & 4.71 & 0.49 & 0.51 & 1313.5 & 0.0022 & 15 \times 15 \\ 9(a) & 2 \mathrm{~d} & 8.94 & 6.3 & 8.94 & 6.3 & 5.7 & 0.40 & 0.474 & 1221 & 0.0015 & 15 \times 15 \\ 10(a) & 20 \mathrm{~m} & 8.49 & 6 & 8.49 & 6 & 5.4 & 0.43 & 0.48 & 1236.3 & 0.0017 & 11.4 \times 7.6 \\ 15(a) & 13 \mathrm{o} & 6.3 & 4.45 & 3.15 & 2.22 & 2 & 0.09 & 0.288 & 741.8 & - & 15 \times 15 \\ 15(c) & 29 \mathrm{j} & 2.76 & 1.94 & 1.38 & 0.97 & 0.843 & 0.0285 & 0.196 & 504.8 & - & 15 \times 15\end{array}$

TABLE 1. Parameters corresponding to the planform structures in the various figures. The first three rows are in the advection regime and the rest in the combined regime.

(a)

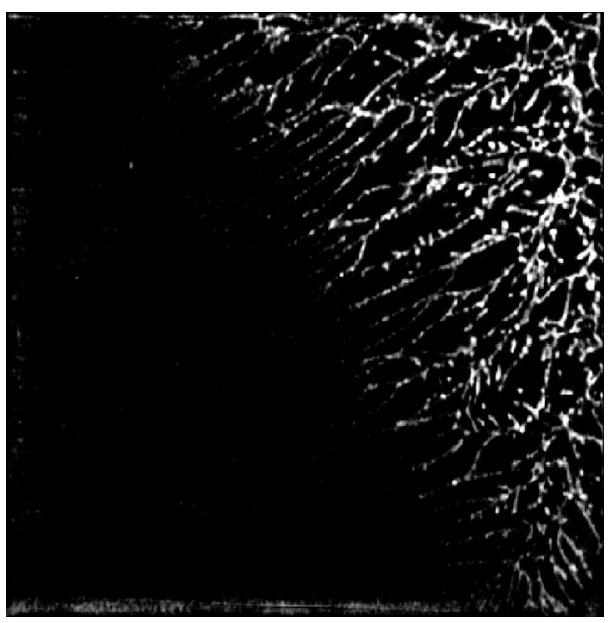

(b)

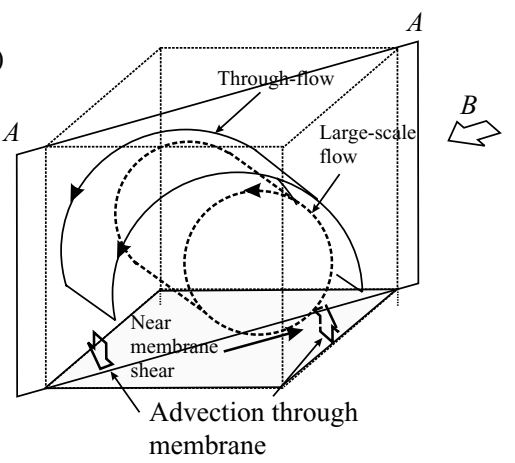

(c)
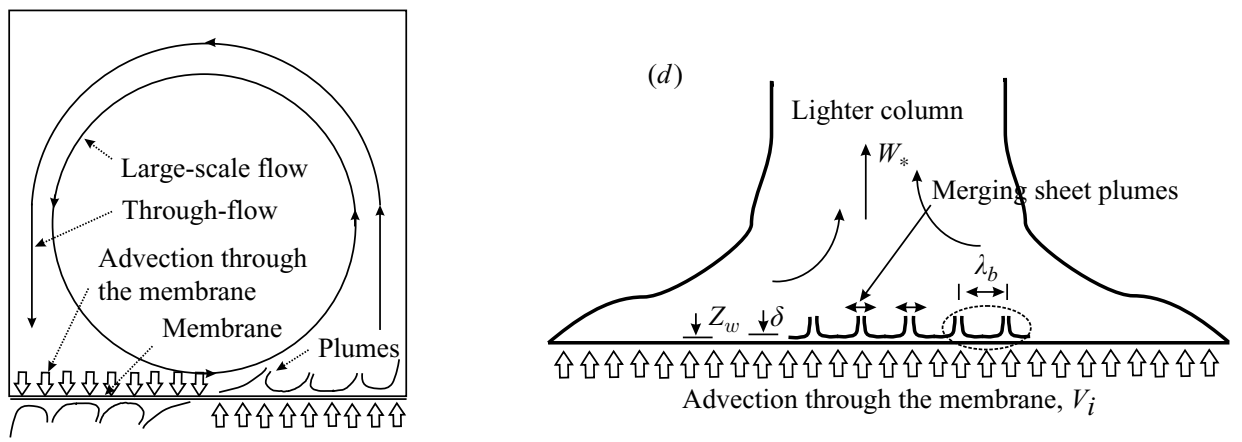

Figure 8. Plume structure in the advection regime. (a) Planform view of the plume structure near the membrane at $R a_{w}=4.71 \times 10^{11}$ for $C_{T}^{0}=10 \mathrm{gl}^{-1}$, after $14.7 \mathrm{~min}$ of the initiation of an experiment. Parameters corresponding to the figure are shown in table 1. (b) Schematic of the flow in the top compartment for $(a)$. The large-scale flow is driven by rising plume columns in the top right of $(a)$, which creates a near-membrane shear from the centre to the top right-hand corner. (c) Schematic of the flow, the plumes and the advection through the membrane in the top compartment in the $A-A$ plane of $(b)$. (d) Schematic of the flow when viewed in the direction $B$ shown in $(b)$. The dashed ellipse in $(d)$ is shown as figure $14(a)$. 
(a)

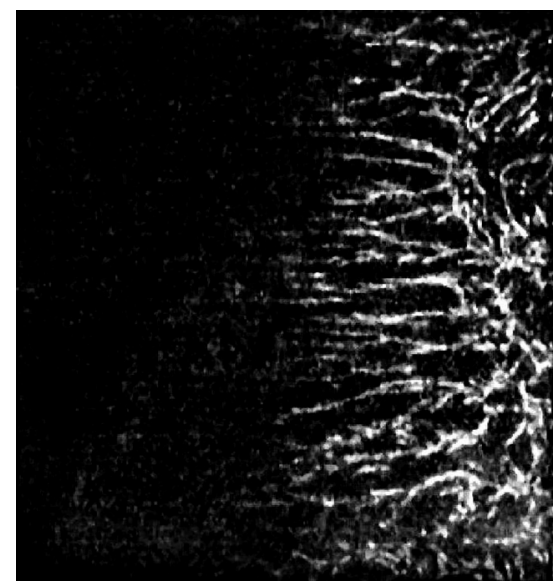

(b)

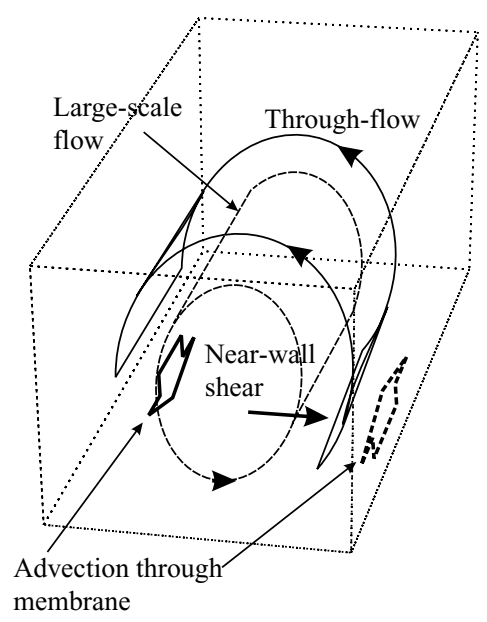

FIGURE 9. (a) Planform plume structure seen due to a parallel-to-walls orientation of the large-scale flow at $R a_{w}=5.7 \times 10^{11}$ in the advection regime. (b) Schematic of the through flow and the large-scale flow in $(a)$. The large-scale flow creates a near membrane mean shear from the left to the right $(\rightarrow)$.

plumes in figure 8(a) are nearly aligned along a diagonal of the tank cross-section. It is well known that a large-scale circulation aligns the near-wall plumes along the mean shear direction in turbulent free convection (PA; Theerthan \& Arakeri 2000). Hence, we could identify the large-scale flow from the alignment of plume lines in the planform and from the side view of the rising plume column.

The bulk flows in the top tank, introduced in $\S 3$, and inferred from the nearmembrane plume structure in figure $8(a)$ are shown in figures $8(b)$ to $8(d)$. In figure $8(a)$, the through-flow is anticlockwise, upward where the plumes are visible above the membrane and downward in the plume-free area above the membrane. The through-flow which impinges on the bottom left-hand part of the figure $8(a)$ prevents the boundary layers from forming on this area, resulting in a plume-free area and advection down through the membrane. The total concentration change would occur in boundary layers on the opposite side of the through-flow impingement (figure $3 b$ ). The large-scale flow in the top tank (see figure $3 a$ also) is anticlockwise, oriented along the diagonal of the test section; a similar large-scale flow would exist in the bottom tank. This large-scale flow is what is referred to as the 'wind' in RBC literature (Niemela et al. 2001; Niemela \& Sreenivasan 2003; Xia, Sun \& Zhou 2003; Funfschilling \& Ahlers 2004). The large-scale flow aligns the near-membrane sheet plumes and sweeps the plume caps toward the corner as shown in figure $8(c)$. The plume caps combine to form rising columns of lighter fluid (figure $8 d$ ), which in turn drive the large-scale flow itself. The strength of the mean large-scale flow in figure $8(a)$ is given by the Deardorff velocity scale $W^{*}=0.51 \mathrm{~cm} \mathrm{~s}^{-1}\left(R e=W^{*} H / v=1314\right)$.

In any experiment, the flux keeps decreasing with a time scale of $t_{q}$, the planform plume structures also change over the same time scale. In experiments with $C_{T}^{0}=10 \mathrm{gl}^{-1}$ the overall alignment and the diagonal division into areas with and without plumes are maintained until a $\Delta C$ of about $7 \mathrm{gl}^{-1}$. The sheet plumes were sometimes observed to be aligned parallel to the walls in the beginning of the advection regime. Figure $9(a)$ shows such an alignment at about 4 min after the start of a $10 \mathrm{gl}^{-1}$ experiment (note that this is not the same as the experiment 
shown in figure $8 a$ ). A schematic of the counterclockwise large-scale flow, causing this plume structure, is shown in figure $9(b)$. This alignment was, however, unstable and soon evolved to the diagonal alignment as in figure $8(a)$. When experiments were started with $C_{T}^{0}=10 \mathrm{gl}^{-1}$, it was difficult to visualize the plume structure below $\Delta C=7 \mathrm{~g}^{-1}$ as the contrast of dye between the plumes and the top-tank solution was not sufficient. Hence, experiments with starting concentrations of $C_{T}^{0}=7 \mathrm{~g}^{-1}$ and $3 \mathrm{~g}^{-1}$ were conducted to study the plume structure in the combined regime; these are discussed in Appendix A. The diffusion regime occurs at very low driving potentials $\left(\Delta C<1.5 \mathrm{~g}^{-1}\right)$; we do not discuss the plume structure of this regime here as they are similar to that in PA.

\subsection{The near-membrane plume dynamics}

As mentioned earlier, the near-wall flow consists of sheet plumes. These sheet plumes are constantly moving and merging. The merging dynamics is determined by the combined influence of mean shear due to the large-scale flow and the entrainment flow field of the adjacent plumes. Two main dynamics are observed near the membrane.

(a) A new plume is initiated in a vacant region as a point, and becomes elongated into a sheet. The elongation is in the direction of the dominant flow in the region.

(b) The sheet plumes move laterally and merge owing to entrainment of fluid between them.

Figure 10(a) shows a close-up view in the shear dominated central region of the plume structure when the planform is similar to that in figure $8(a)$. We observe main sheet plumes, aligned along the direction of shear (white arrow), with small branches at an angle; the structure has a dendritic appearance. Figures $10(b)$ to $10(\mathrm{~g})$ show the dynamics of formation of these dendritic structures. In figure $10(b)$, two main sheet plumes indicated by $(A)$, separated by about $0.16 \mathrm{~cm}$ move laterally and merge in about $4 \mathrm{~s}$. This merger is due to the local entrainment flow (double-headed white arrow), perpendicular to the plume lines. A plume is initiated as a point at (B) in the vacant area left by the merger of the main sheet plumes. This plume becomes elongated in the direction of the resultant of the mean shear and the entrainment flow, to join the main plumes at an angle (figure 10d). The small plume at (B) also moves laterally toward the main plume owing to the entrainment flow created by the main plume (compare the orientation of plume at (B) in figures $10 \mathrm{c}$ and $10 \mathrm{~g}$ ). Other small plumes initiated in the nearby vacant areas undergo the same dynamics (figure $10 e-g$ ) to give rise to a main sheet plume with small dendritic plume branches at an angle. There is a flow of lighter fluid along the main sheet plume owing to the mean shear. Owing to this flow, the whole structure also moves along the direction of the mean shear. This motion can be seen clearly by observing the plume segment at $\mathbb{C}$ in figure $10(b)$ passing out of view with time. Note that the plume dynamics has a much smaller time scale of $\sim 7 \mathrm{~s}$ than the time scale of $\sim 47 \mathrm{~s}$ of the large-scale flow. See movie 1 (available with the online version of the paper) for the dynamics corresponding to figure $10(a)$.

The two main features, of elongation and merging, remain similar to the observations of PA for convection in the diffusion regime; the common dynamics was also shown in Puthenveettil et al. (2005). As we shall see below, the advection velocities through the membrane are much smaller than the near-membrane velocity scales. Hence, the common dynamics is not surprising as it is the gravitational instability of the boundary layers that results in the formation of plumes (Theerthan \& Arakeri 2000; PA) in both the regimes. However, in the strong shear areas, the structures in the advection regime in the present study look quite different from the plume structures in PA. The present study has larger flux, which gives rise to stronger mean 
(a)

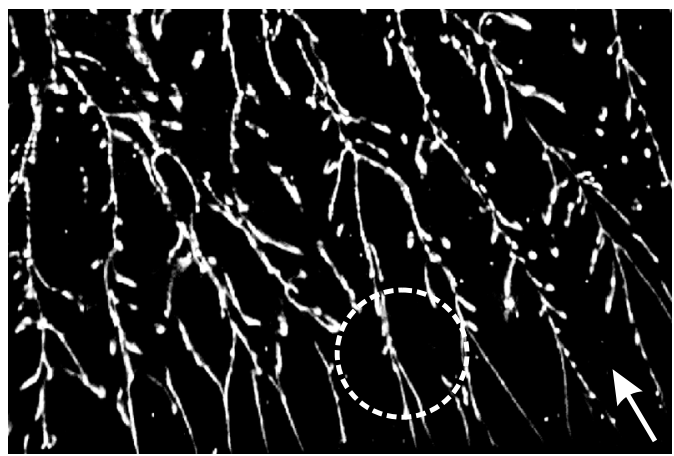

(b)

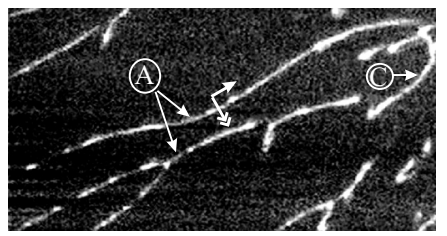

(e)

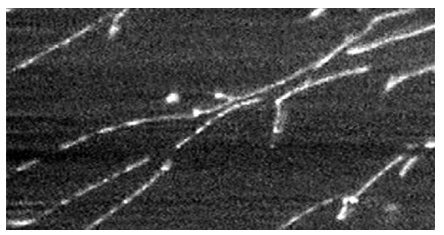

(c)

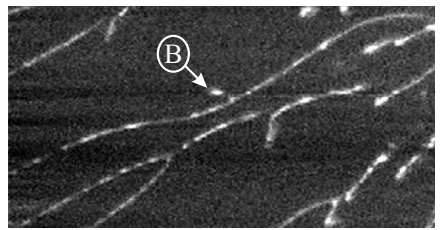

$(f)$

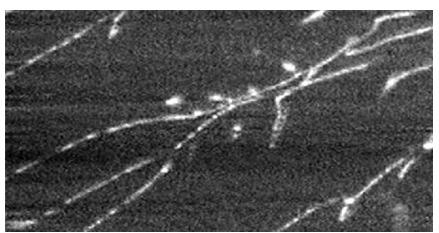

(d)

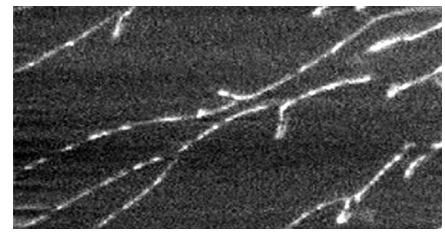

(g)

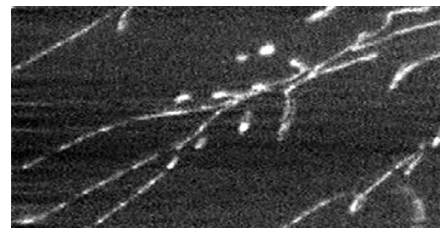

FIGURE 10. (a) Enlarged planform view of the central part of the plume structure of figure $8(a)$ showing the dendritic structure. The white arrow indicates the direction of mean shear due to large-scale flow. The parameters corresponding to the figure are shown in table $1 .(b)-(\mathrm{g})$ Dynamics in the region indicated by the dashed circle in $(a)$. Images from $(b)$ to $(e)$ are $1 \mathrm{~s}$ apart. Images from $(e)$ to $(g)$ are $0.5 \mathrm{~s}$ apart. The main sheet plumes, indicated by $(\mathbb{A}$, merge. At (B) a plume is initiated. The double-headed white arrow indicates the local entrainment flow field direction at (B); the arrow at right angles to that indicates the direction of mean shear due to large-scale flow at (B). The part indicated by $(\mathrm{C}$ moves out of view owing to the motion of the whole plume structure along the mean shear direction. The direction of gravity in all the images is perpendicular to the plane of the figure and downward. The size of images in $(b)$ to $(g)$ is $2.82 \mathrm{~cm} \times 1.47 \mathrm{~cm}$. See movie 1 for the dynamics corresponding to $(a)$.

shear and entrainment flows, resulting in faster merging. The stronger entrainment flows in the present study seem to pull the smaller plumes toward the main plumes resulting in the dendritic structure.

\subsection{Phenomenology of the cubic scaling}

The planform in figure $8(a)$ corresponds to the advection regime where $q \sim \Delta C^{3}$ (figure $6 a$ ). The impingement of the through-flow on one side of the membrane prevents formation of boundary layers on that side and the total concentration change would occur in boundary layers on the other side of the membrane (Appendix D). All the transport across the membrane is by advection, none by diffusion. It is shown below that the cubic scaling can be explained by assuming a large-scale flow, having the strength of the Deardorff velocity scale, driving through the membrane a flow which obeys the D'Arcy law. 
(a)

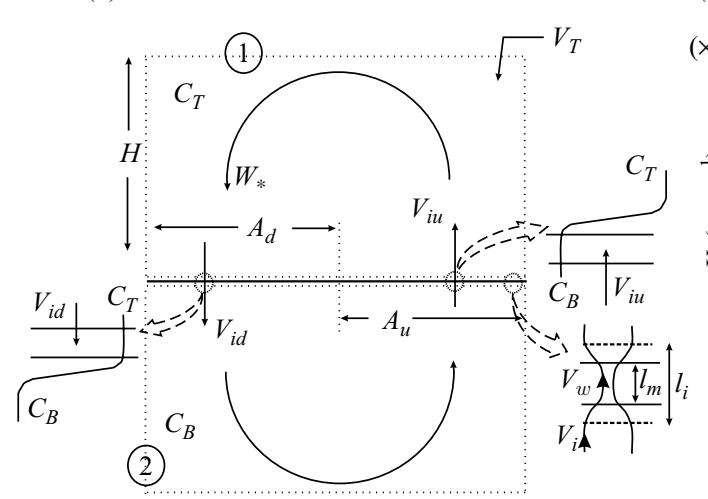

(b) 4

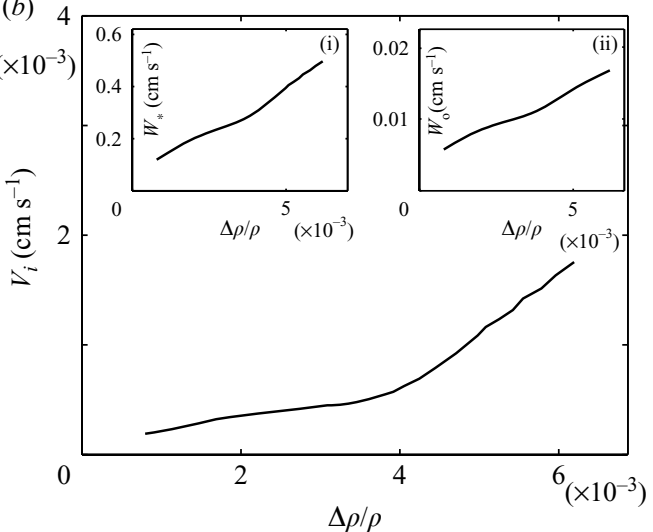

FIGURE 11. (a) Schematic of control volume, variables and flow for the model to explain the cubic flux scaling. (1) and (2) denote the control volumes of the top and the bottom tanks, respectively. The concentration profiles on the up-flow and the down-flow parts of the membrane are shown on the right and the left of the figure. $(b)$ The dependence of the advection velocity $V_{i}$ on $\Delta \rho / \rho$, estimated by (5.5), for the $C_{T}^{0}=10 \mathrm{~g}^{-1}$ experiment in figure 6 . Inset (i) shows the variation of the bulk velocity scale $W_{*},(2.6)$. Inset (ii) shows the variation of the near-membrane velocity scale $W_{o},(5.6)$.

Let the large-scale flow velocity be proportional to the Deardorff velocity scale $W^{*}$, given by (2.6); $V_{L S}=C_{1} W^{*}$, where $V_{L S}$ is the large-scale flow velocity and $C_{1}$ is a constant. The Reynolds number corresponding to the large-scale flow in the advection regime is about 1300 (table 1). This large-scale circulation impinging on the membrane creates a pressure difference $\Delta P$ that drives a flow across the membrane;

$$
\Delta P=\frac{1}{2} \rho V_{L S}^{2}=\frac{1}{2} C_{1}^{2} \rho(g \beta q H)^{2 / 3} .
$$

The balance of driving pressure and viscous resistance given by D'Arcy's law gives the average advection velocity $V_{i}$ at a plane slightly away from the mesh surface (see detail on the right-hand side of figure 11a) as,

$$
V_{i}=\frac{\kappa}{\mu} \frac{\Delta P}{l_{i}}=\frac{C_{1}^{2} \kappa}{2 v l_{i}}(g \beta q H)^{2 / 3},
$$

where $\kappa$ is proportional to the membrane permeability, and $l_{i}$ is proportional to the thickness of the membrane, $l_{m}$.

To obtain the flux scaling, we now express $V_{i}$ in (5.2) in terms of $\Delta C$ by mass balance of the top-tank control volume. Consider the case when the bottom part of the top-tank control volume has upward flow in half the area and downward flow in the remaining half (figure 11a). The area of the test-section cross-section $A=A_{u}+A_{d}$, where $A_{u}$ and $A_{d}$ are the upward and the downward advection areas, respectively. Mass conservation of the control volume (1) in figure 11(a) gives the rate of change of mass of salt in the top tank,

$$
V_{T} \frac{\mathrm{d} C_{T}}{\mathrm{~d} t}=C_{B} V_{i u} A_{u}-C_{T} V_{i d} A_{d},
$$

where the symbols are as shown in figure 11(a). Diffusive transport across the mesh has been neglected in the above relation. The incompressibility assumption gives

$$
V_{i d} A_{d}=V_{i u} A_{u} .
$$


Using (5.4) in (5.3) and if $A_{u}=A_{d}=A / 2$, then as $q=-H\left(\mathrm{~d} C_{T} / \mathrm{d} t\right)$ from (2.3), we obtain the advection velocity as,

$$
V_{i u}=V_{i d}=V_{i}=\frac{2 q}{\Delta C} .
$$

We use (5.5) to estimate $V_{i}$, the average advection velocity in each half of the membrane in the advection regime.

Figure 11(b) shows the variation of $V_{i}$ in a $C_{T}^{0}=10 \mathrm{gl}^{-1}$ experiment, as estimated from the data in figure 6(a) using (5.5). (5.5) is derived assuming that the diffusive transport is absent; thus the estimate of $V_{i}$ will be valid only in the advection regime at $\Delta \rho / \rho>4.5 \times 10^{-3}$ where $q \sim \Delta C^{3}$. Note that the advection velocity is small compared to the velocity scales in turbulent-free convection. The Deardorff velocity scale is about 300 times the advection velocity at the beginning of the advection regime (inset (i) in figure 11b). Townsend's near-wall velocity scale (Townsend 1959) in turbulent convection

$$
W_{o} \sim(g \beta q D)^{1 / 4},
$$

shown as inset (ii) in figure $11(b)$, is about ten times $V_{i}$.

By substituting (5.2) for $V_{i}$ in (5.5) and simplifying, we obtain the cubic dependence of flux on $\Delta C$ as

$$
q=\left(\frac{C_{1}^{2} \kappa}{4 v l_{i}}\right)^{3}(g \beta H)^{2} \Delta C^{3} .
$$

Equation (5.7) can be written in dimensionless terms as the Nusselt number in the presence of advection as

$$
N u_{b}=C_{2} \varkappa R a_{\kappa} G r=\varkappa_{1} \frac{R a^{2}}{S c},
$$

where, $C_{2}=\left(C_{1} / 2\right)^{6}$ is a constant, $\varkappa=\kappa^{2} / l_{i}^{4}$ is a dimensionless membrane parameter, $\varkappa_{1}=C_{2} \chi \kappa l_{i} / H^{3}, \quad R a_{\kappa}=g \beta \Delta C \kappa l_{i} /(v D)$ is the D'Arcy Rayleigh number of the membrane, $G r=g \beta \Delta C H^{3} / \nu^{2}$ is the Grashoff number of the fluid layer, and $R a=G r S c$. The Rayleigh-number dependence predicted by (5.8) matches well with the experimental results shown in figure $6(b)$.

The results reported in the present study cover a limited range of $R a$ (about $3 \times 10^{11}$ to $6 \times 10^{11}$ ) in the advection regime. Clearly, more experiments over a larger range of $R a$ are required to prove conclusively the $\Delta C^{3}$ scaling and the validity of the proposed model. At this stage, the model may be treated as a possible explanation for the observed flux scaling in the advection regime. However, since the physical mechanism of transport across the membrane changes over shorter ranges of $R a$ in the present arrangement, it may not be realistic to expect scaling laws over large $R a$ ranges as in RBC. We expect the $R a^{2}$ scaling in the advection regime to hold as long as:

(a) the advection velocity in the membrane is smaller than $W_{o}(5.6)$ and

(b) the inertial and the diffusive effects are negligible in the transport through the membrane.

If condition $(a)$ is not valid, the boundary layers could be blown away by the advection velocity. Since the value of $W_{o}$ is much smaller than the velocity at which inertial effects become predominant in the membrane, condition $(a)$ will be violated first when $R a$ is increased. The second assumption of no diffusive flux across the membrane is 


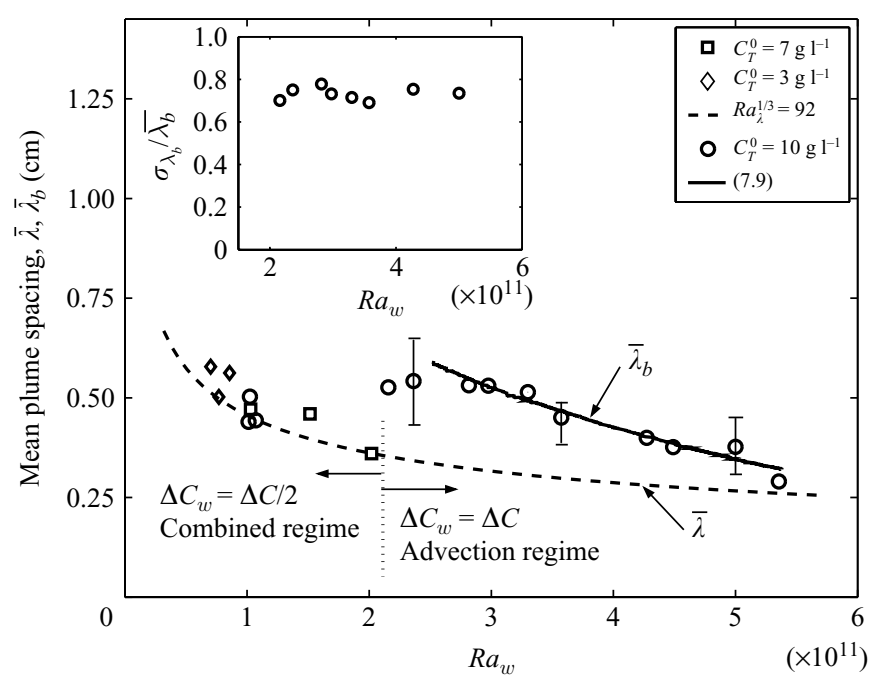

Figure 12. Variation of the mean plume spacing with $R a_{w}$ for $C_{T}^{0}=10 \mathrm{gl}^{-1}, C_{T}^{0}=7 \mathrm{gl}^{-1}$ and $C_{T}^{0}=3 \mathrm{gl}^{-1}$ experiments. The solid line is (7.9) and the dashed line is (6.1). The inset shows the normalized variance of plume spacings in the advection regime.

justified in Appendix D. By using $W_{o}$ instead of $V_{i}$ in (5.2) we can estimate

$$
R a_{t r}=\left(\frac{2^{14}}{C_{1}^{18}}\right)^{1 / 5}\left(\frac{l_{i} H}{\kappa}\right)^{9 / 5} S c^{4 / 5}
$$

as the transition $R a$ at which the $R a^{2}$ scaling could change. Using $5 \geqslant C_{1} \geqslant 0.5$ (order of 1 ), for the present membrane properties, we obtain $6.4 \times 10^{12} \leqslant R a_{t r} \leqslant 2.54 \times 10^{16}$. If we take the lower value of $R a_{t r}$, it will imply that the whole range of $R a^{2}$ scaling itself could be only about a decade in our experiments.

\section{Plume spacing distribution}

The sheet plumes are formed owing to the boundary layers between them becoming unstable along lines (Spangenberg \& Rowland 1961; Kerr 1996; Husar \& Sparrow 1968; Sparrow \& Husar 1969; Theerthan \& Arakeri 2000). The spacings between adjacent plume lines show the distance at which the boundary layers become unstable. We study these spacings to gain insights into the nature of the near-membrane boundary layers. The plume spacings are measured from the planform images using adjacent plume line coordinates input into a program by mouse clicks. The measurements are made as normal as possible to the plume lines all over the planform to cover the whole range of spacings. The sample size of the measurements is dependent on the number of plume lines, which increased with increase in $R a_{w}$. For planforms similar to that in figure $8(a)$ the sample size is about 700, while for planforms toward the end of the advection regime the sample size is about 350 . The statistics are improved by taking the spacings measured from about three images a short time apart so that the images are almost at the same $R a_{w}$. The outliers in the data are removed by the criterion suggested by Frank \& Althoen (2002) for skewed distributions.

\subsection{Mean spacing variation}

Figure 12 shows the variation of the mean plume spacings with $R a_{w}$ for the $C_{T}^{0}=$ $10 \mathrm{gl}^{-1}, 7 \mathrm{~g}^{-1}$, and $3 \mathrm{gl}^{-1}$ experiments. The Rayleigh number in figure 12 is calculated 
based on the appropriate $\Delta C_{w}$ for each of the regimes; $\Delta C_{w}=\Delta C$ for the advection regime and $\Delta C_{w}=\Delta C / 2$ for the combined regime. In all the experiments, the mean plume spacings decrease with increase in $R a_{w}$.

The theoretical prediction for the diffusion regime at $S c \sim 600$ (see PA),

$$
R a_{\lambda}^{1 / 3}=\bar{\lambda} / Z_{w} \simeq 92
$$

is plotted in the figure as the dashed line. Here, $R a_{\lambda}=g \beta \Delta C_{w} \bar{\lambda}^{3} /(v D)$ is the Rayleigh number based on the mean plume spacing $\bar{\lambda}$ in the diffusion regime. It is seen that the plume spacings in the combined regime for the $C_{T}^{0}=10 \mathrm{gl}^{-1}, 7 \mathrm{gl}^{-1}$ and $3 \mathrm{gl}^{-1}$ experiments, which gave $q \sim(\Delta C / 2)^{4 / 3}$ scaling, match the prediction of (6.1) when $\Delta C_{w}=\Delta C / 2$. In other words, the dependence of the mean plume spacing on $R a_{w}$ is the same in the combined and the diffusion regimes. As (6.1) assumes that the nearmembrane boundary layers are laminar natural convection boundary layers, we expect that the near-membrane boundary layers in the combined regime have the character of laminar natural convection boundary layers with no wall normal advection.

The mean plume spacings in the advection regime, $\overline{\lambda_{b}}$ for the $C_{T}^{0}=10 \mathrm{gl}^{-1}$ experiments, is seen as a distinct curve above the $R a_{\lambda}^{1 / 3} \simeq 92$ curve. Advection increases the mean plume spacing from that in the combined regime for the present range of Rayleigh numbers and advection velocities. The Rayleigh-number dependence in the advection regime is also different from that in the combined regime. An analysis which explains these differences is proposed in $\S 7$. The inset in figure 12 shows the variation of the normalized standard deviation of plume spacings $\sigma_{\lambda_{b}} / \overline{\lambda_{b}}$ with $R a_{w}$ in the advection regime. As $\sigma_{\lambda_{b}} / \overline{\lambda_{b}} \approx 0.7$, the variance increases proportionally to the mean plume spacing. An increase in $R a_{w}$ results in a greater number of closely spaced plumes (lower $\overline{\lambda_{b}}$ ). The variation between the plume spacings also reduces with increase in $R a_{w}$ (lower $\sigma_{\lambda_{b}}$ ). Similar conclusions were reached for plume spacings in the diffusion regime by PA.

\subsection{Spacing statistics}

Figure 13(a) shows the histogram of plume spacings, normalized by the mean plume spacing, at the beginning of the advection regime at $R a_{w}=5 \times 10^{11}$. The data for the plot is taken from three images about $30 \mathrm{~s}$ apart so that all the images are at approximately the same $R a_{w}$. The planforms corresponding to this histogram are as in figure $8(a)$. The spacings show a skewed distribution with a long tail. As shown in the inset of figure $13(a)$, the probability density function (PDF) of the logarithm of the plume spacings, in the standardized form $z=\left(\ln \left(\lambda_{b} / \overline{\lambda_{b}}\right)-\overline{\ln \left(\lambda_{b} / \overline{\lambda_{b}}\right)}\right) / \sigma_{\ln \left(\lambda_{b} / \overline{\lambda_{b}}\right.}$, matches well with the standard normal PDF given by $\exp \left(-\mathrm{z}^{2} / 2\right) / \sqrt{2 \pi}$. Here, the overbar indicates mean and $\sigma$ indicates standard deviation. The distribution of spacings can hence be approximated by a log-normal distribution. Even though the mean and the variance increased with decrease in $R a_{w}$ (figure 12), the distribution remained log normal until the end of the advection regime at $R a_{w} \approx 2 \times 10^{11}$. This common form of PDF allows us to combine the plume spacing data from all the images in the advection regime. The PDF from the combined data of 4352 plume spacings is shown in figure 13(b); the standard log-normal distribution fits the data well. We conclude that the PDF of plume spacings in the advection regime of our experiments can be approximated by the standard log-normal function for $2 \times 10^{11}<R a_{w}<5 \times 10^{11}$ at $S c=600$. The variation of the standardizing variables $\overline{\ln \left(\lambda_{b} / \overline{\lambda_{b}}\right)}$ and $\sigma_{\ln \left(\lambda_{b} / \overline{\lambda_{b}}\right)}$ with $R a_{w}$ is shown in insets (i) and (ii) of figure 13(b), respectively. The plume spacing distribution in the advection regime of the present experiments is completely described 

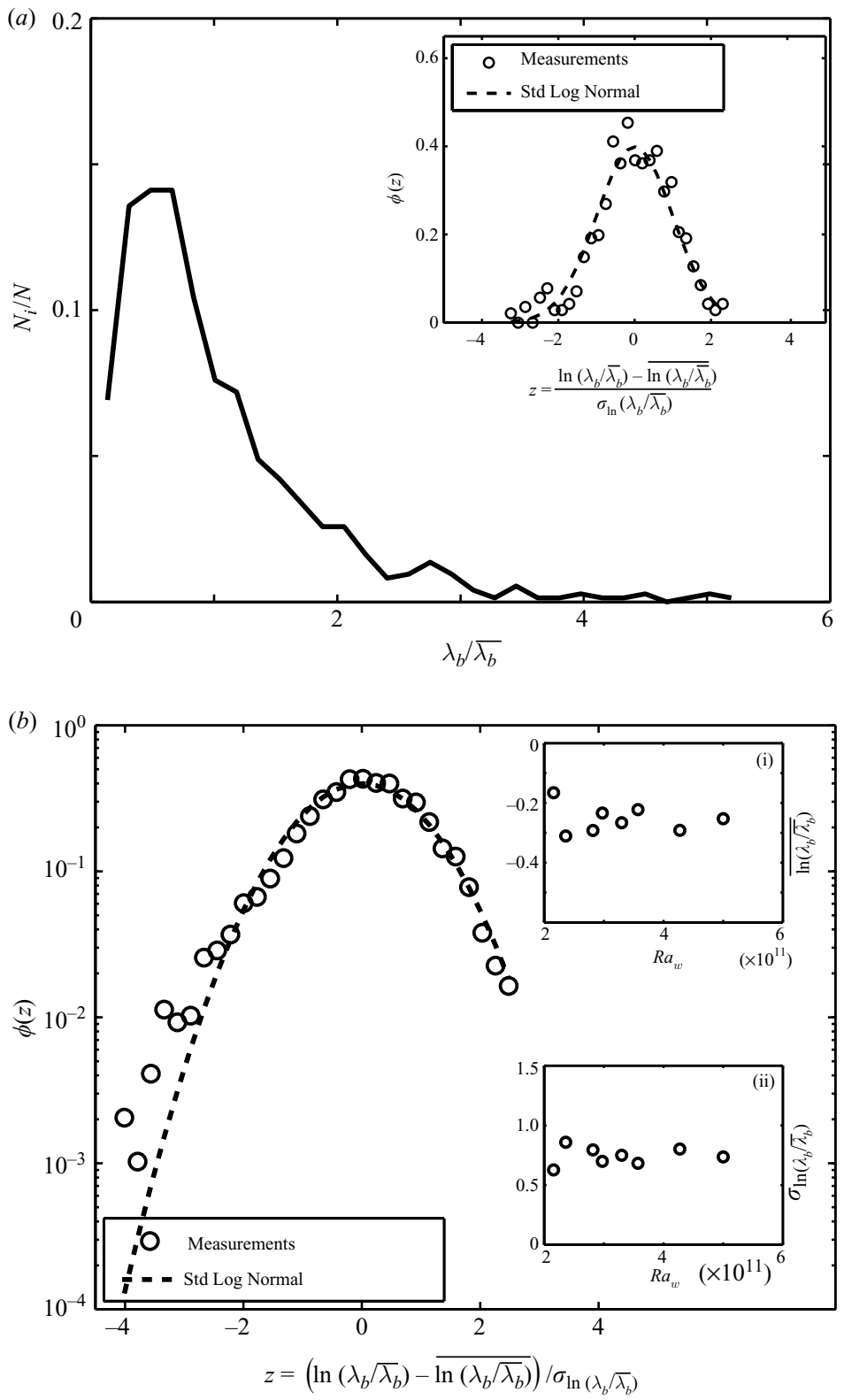

FIGURE 13. (a) The histogram of plume spacings in the beginning of the advection regime at $R a_{w}=5 \times 10^{11}$. The inset shows the probability density function of the logarithm of plume spacings in the standardized form. (b) The common log-normal probability density function of the plume spacings in the advection regime obtained from the combined data of all the images in the advection regime. Insets (i) and (ii) shows the variation of the standardizing parameters $\overline{\ln \left(\lambda_{b} / \overline{\lambda_{b}}\right)}$ and $\sigma_{\ln \left(\lambda_{b} / \overline{\lambda_{b}}\right)}$, respectively, with $R a_{w}$.

by figure $13(b)$. Similar results of a common log-normal PDF of plume spacings were obtained for the diffusion regime by PA. Puthenveettil et al. (2005) had also shown that the plume structures for the advection and the diffusion regimes had a common multifractal spectrum; it was proposed to be due to the common dynamics underlying 


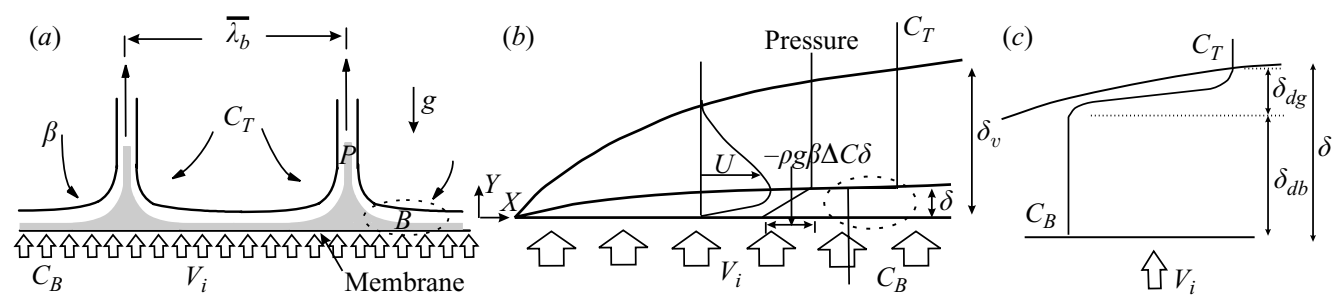

FIGURE 14. Schematic of the model for the mean plume spacings near the membrane in the advection regime. The dashed ellipse in $(a)$ is shown as $(b)$, the dashed ellipse in $(b)$ is shown as (c). (a) The model as a regular array of two high-Schmidt-number laminar natural convection boundary layers with normal advection at the membrane surface giving rise to plumes spaced $\overline{\lambda_{b}}$ apart. The lines show the edge of the velocity boundary layer and the shaded regions show the species boundary layer and plume. $B$ indicates boundary layer and $P$ indicates plume. (b) Details of the boundary-layer. $\delta$ is the species boundary-layer thickness. $\delta_{v}$ is the velocity boundary-layer thickness. $(c)$ The concentration profile in the species boundary layer.

the formation of these structures. This proposition gains more value as the main features of the near-membrane dynamics in $\$ 5.2$, namely, the initiation, elongation and merging of sheet plumes, is the same as that observed by PA for the diffusion regime. Further quantification of the near-membrane dynamics must be conducted to make stronger conclusions.

\section{A model for the mean plume spacings in the advection regime}

We have seen that in the advection regime, the sheet plumes have a larger spacing and a different dependence on $R a_{w}$ compared to the diffusion regime in PA; we now propose a model which explains this difference. Since sheet plumes are the results of the instability of the boundary layers between them, we hypothesize that the near-membrane boundary layers between the sheet plumes are laminar natural convection boundary layers. Rigorous justifications for the assumption of laminar natural convection boundary layer were discussed in Theerthan \& Arakeri (1998) and PA. In the advection regime, these boundary layers will be forced by a wall normal flow. At some thickness of these boundary layers, gravitational instability causes the boundary layer fluid to turn upward to form a sheet plume. We approximate the observed plume structure as a regular array of plume sheets, spaced apart by the mean spacing $\overline{\lambda_{b}}$, (figure 14a). We then obtain an expression for the species boundarylayer thickness from an order of magnitude analysis of the integral boundary-layer equations at high Schmidt number. An expression for the mean plume spacing is then obtained using the gravitational instability condition of the boundary layer.

\subsection{Order of magnitude analysis of integral equations}

Figure 14(b) shows a schematic of the steady laminar natural convection boundary layer at high $S c$ on a horizontal surface with a constant blowing velocity $V_{i}$. The relevant integral equations for these boundary layers are given in Appendix B. As an approximation of the actual concentration profile shown in figure 14(c), we assume the species boundary layer to be of constant concentration $C_{B}$ throughout its thickness; the assumption is justified in Appendix $C$. The following analysis is not valid for small advection velocities where there will be appreciable concentration gradients through a major portion of the species boundary layer. Under the uniform concentration approximation, $C_{T}-C(Y)=\Delta C$. Then, using (B11), the integral $X$-momentum 
equation (B 9) reduces to

$$
\frac{\mathrm{d}}{\mathrm{d} X} \int_{0}^{\delta_{v}(X)} U^{2} \mathrm{~d} Y-\frac{1}{2} g \beta \Delta C \frac{\mathrm{d}}{\mathrm{d} X} \delta^{2}+\left.v \frac{\partial U}{\partial Y}\right|_{Y=0}=0,
$$

The equation expresses a balance of the horizontal momentum flux with the horizontal pressure gradient and the wall shear stress. Here, $X$ and $Y$ denote the horizontal and the vertical directions, $U$ and $V$ the velocities in these directions, $\delta$ the species boundary-layer thickness and $\delta_{v}$ the velocity boundary-layer thickness. The integral species equation $(\mathrm{B} 10)$ reduces to

$$
\frac{\mathrm{d}}{\mathrm{d} X} \int_{0}^{\delta(X)} U \mathrm{~d} Y=V_{i}
$$

An order of magnitude balance of (7.2) gives

$$
\frac{U \delta}{X} \sim V_{i}
$$

The order of magnitude balance of (7.1), division by $g \beta \Delta C \delta^{2} / X$ and substituting for $U$ from (7.3) gives

$$
\frac{V_{i}^{2} X^{2} \delta_{v}}{g \beta \Delta C \delta^{4}}+\frac{V_{i} X^{2} v}{g \beta \Delta C \delta^{4}} \sim 1 .
$$

The first term is the ratio of inertial effects to the motion pressure while the second term is the ratio of viscous effects to the motion pressure.

\subsection{Estimation of plume spacing from the near-membrane scales}

The near-membrane scales are decided by the predominant balance between the terms in (7.4). The ratio of the first and second terms in (7.4) is $R e_{v}=V_{i} \delta_{v} / \nu$, a Reynolds number. When $R e_{v} \ll 1$, as the first term can be neglected compared to the second, the predominant balance in the boundary layer is between the driving motion pressure gradient and the viscous resistance. For typical experimental values at the beginning of the advection regime $\left(C_{T}^{0}=10 \mathrm{~g}^{-1}, V_{i}=0.0018 \mathrm{~cm} \mathrm{~s}^{-1}\right.$, $\left.v=0.0089 \mathrm{~cm}^{2} \mathrm{~s}^{-1}, \delta \sim 0.02 \mathrm{~cm}\right), R e_{v}=0.0033$. The experimental results fall in the range of $R e_{v} \ll 1$. The species boundary-layer thickness, for the advection velocities encountered in the present experiments, hence scales as,

$$
\delta \sim\left(\frac{V_{i} \nu X^{2}}{g \beta \Delta C}\right)^{1 / 4}
$$

From (7.3), the horizontal velocity in the boundary layer scales as,

$$
U \sim\left(\frac{V_{i}^{3} X^{2} g \beta \Delta C}{v}\right)^{1 / 4}
$$

The expressions (7.5) and (7.6) can also be written as,

$$
\frac{\delta}{X} \sim \sqrt{\frac{\zeta}{X}}, \quad \frac{U}{V_{i}} \sim \sqrt{\frac{X}{\zeta}},
$$

where $\zeta=\sqrt{V_{i} \nu /(g \beta \Delta C)}$ is a boundary-layer length scale in the presence of advection.

To estimate the plume spacing, we need a stability condition for natural convection boundary layers with wall normal advection. To our knowledge, no theoretical estimate of the stability of such boundary layers is available. However, it is known 
that in natural convection boundary layers over horizontal surfaces, the gravitational mode of instability is usually predominant, resulting in the stability condition $R a_{\delta} \approx 1000$ (Sparrow \& Husar 1969; Rotem \& Classen 1969). We expect the same instability mechanism to be active in the boundary layers in the advection regime for the present advection velocities. As the near-membrane transport is by advection and not by diffusion in the present case, we expect the stability condition for highSchmidt-number boundary layers with wall normal advection to be

$$
G r_{\delta}=\frac{g \beta \Delta C \delta^{3}}{v^{2}}=K
$$

where $G r_{\delta}$ is the Grashoff number based on the species boundary-layer thickness and $K$ is a constant. Equating the expression for the boundary-layer thickness, obtained from (7.8), to (7.5) and replacing $X$ by $\overline{\lambda_{b}} / 2$, we obtain

$$
\overline{\lambda_{b}} \sim 2 K^{2 / 3} S c^{1 / 6} \sqrt{Z_{w} Z_{V_{i}}}
$$

where $Z_{w}$ is given by (4.2) and

$$
Z_{V_{i}}=v / V_{i}
$$

is a length scale due to the advection. In the diffusion regime, the mean plume spacing is proportional to the wall length scale in turbulent convecton $\left(\bar{\lambda} \simeq 92 Z_{w}\right)$. The presence of an advection makes the mean plume spacings proportional to the square root of the product of $Z_{w}$ with the length scale due to the advection, $Z_{V_{i}}$. The relative magnitudes of these two length scales decide whether the mean plume spacings in the advection regime are larger than those in the diffusion case.

Equation (7.9) can be expressed as an expression for the dimensionless plume spacing in the presence of advection as

$$
R a_{\lambda_{b}}^{1 / 3}=\overline{\lambda_{b}} / Z_{w}=2 K^{2 / 3} R a_{w}^{1 / 6} \sqrt{\frac{Z_{V_{i}}}{H}} S c^{1 / 6},
$$

where $R a_{\lambda_{b}}=g \beta \Delta C_{w} \bar{\lambda}_{b}^{3} /(v \alpha)$. The equivalent plume spacing expression $\overline{\lambda_{b}}=R a_{\lambda_{b}}^{1 / 3} Z_{w}$, is plotted as the solid line in figure 12 . We have used $K=0.325$ to fit the data. The curve matches the trend of the plume spacings in the advection regime.

The variation of the advection length scale $Z_{V_{i}}$ in the advection regime can be calculated from figure $11(b)$ to be between 5 and $15 \mathrm{~cm}$. As $Z_{V_{i}} \gg Z_{w}$ for the present experimental conditions, the plume spacings in the presence of advection are larger than in the corresponding diffusion case. The plume spacings would decrease with advection velocity as $1 / \sqrt{V_{i}}$. As $Z_{w} \sim 1 / \Delta C^{1 / 3}$ and $Z_{V_{i}} \sim 1 / V_{i} \sim 1 / \Delta C^{2}$, for $\Delta C^{3}$ scaling of flux, $\lambda_{b} / \lambda \sim \sqrt{Z_{V_{i}} / Z_{w}} \sim \Delta C^{-5 / 6}$. Therefore, at larger $\Delta C$ (or larger flux), we could expect that the plume spacings in the advection regime would be lower than for the diffusion case. More experiments are required to verify these predictions.

\section{Conclusions}

In the present study, we investigated high-Rayleigh-number, high-Schmidt-number convection driven by unstable concentration differences $(\Delta C)$ across a porous membrane which allows a flow through it. The concentration difference was maintained by a layer of brine over a layer of distilled water, separated by the horizontal membrane, in square-cross-section tanks. The situation is an intermediate case between two limits: (a) Rayleigh-Taylor instability in the absence of a membrane (Young 
et al. 2001); and (b) pure diffusion across a membrane with very small pores (Puthenveettil \& Arakeri 2005). The following three regimes of convection, based on the dominant mode of transport across the membrane, were detected.

(a) An advection regime, where the flux scaled as $\Delta C^{3}$ at higher driving potentials; the transport of salt across the membrane is predominantly by advection.

(b) A diffusion-dominated regime where the flux scaled as $\Delta C_{w}^{4 / 3}$ at low driving potentials, where $\Delta C_{w}$ is the concentration difference between the membrane surface and the bulk; the transport of salt across the membrane is predominantly by diffusion.

(c) A combined regime where the flux scaled as $(\Delta C / 2)^{4 / 3}$ for driving potentials intermediate to that in the advection regime and the diffusion regime.

In all the regimes, unstable layers of fluid were formed near the membrane. These layers gave rise to sheet plumes, the sheet plumes combined to form plume columns that caused the large-scale flow.

In the advection regime, a slow overturning through-flow was present across the membrane, dividing the membrane surface into two equal areas, one with up flow and the other with down flow. The top side of the membrane had sheet plumes in the up-flow region and no plumes in the down-flow region (figure 8a). A layer of lighter (resp. heavier) fluid was formed on the top (resp. bottom) side of the membrane in the up (resp. down) flow region, which became unstable to give rise to sheet plumes. The transport across the membrane was dominated by advection. The advection velocities through the membrane were ten times smaller than the near-wall velocity scale in turbulent free convection (figure $11 b$ ). A phenomenology based on the above understanding retrieved the observed cubic flux scaling (equation (5.7), figure $6 a$ ). In addition to the through-flow, there were large-scale circulations in the top and the bottom tanks which created shear near the membrane. The mean shear due to the large-scale flow aligned the near-membrane sheet plumes. The plume structure appeared dendritic owing to the combined effect of the mean shear of large-scale flow and the entrainment flow of the adjacent plumes (figure 10a).

The mean plume spacings in the advection regime showed larger values and different Rayleigh-number dependence compared to that in the diffusion regime. Increase in Rayleigh number resulted in a larger number of closely and regularly spaced sheet plumes (figure 12). The plume spacings showed a common log-normal distribution in the advection regime (figure 13). Using integral equations of high-Schmidt-number laminar natural convection boundary layers with a wall normal flow, we derived the expression for the concentration boundary-layer thickness (equation (7.5)). Assuming that the gravitational instability condition is valid, an expression for the mean plume spacing in the advection regime was obtained (equation (7.9)); the predictions matched the measurements. The mean plume spacings were seen to be proportional to the square root of the product of the near-wall length scale of turbulent convection, $Z_{w}$ (equation (4.2)), and a length scale due to the advection velocity, $Z_{V_{i}}$ (equation (7.10)).

In the combined regime, the magnitude of the dimensionless flux calculated based on $\Delta C / 2$ matched with the diffusion values; the flux scaled as $\Delta C / 2)^{4 / 3}$ (figure $7 b$ ). Based on these results, we inferred that the boundary layers retain their diffusive nature for the weak advection velocities at the membrane surface encountered in this regime.

The present study has not investigated the effects of the membrane properties on the range of the three regimes. Further studies with different membranes must be conducted to investigate the $R a$ range of the flux scalings observed here. 
(a)

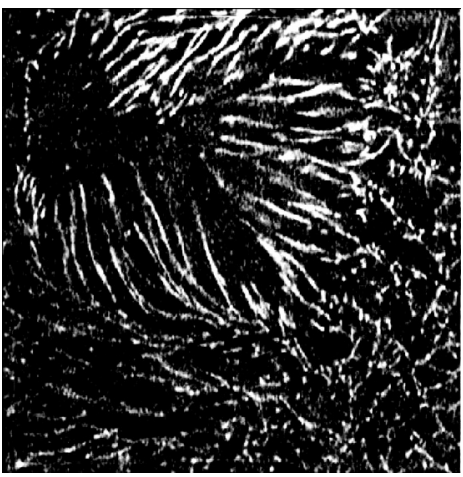

(c)

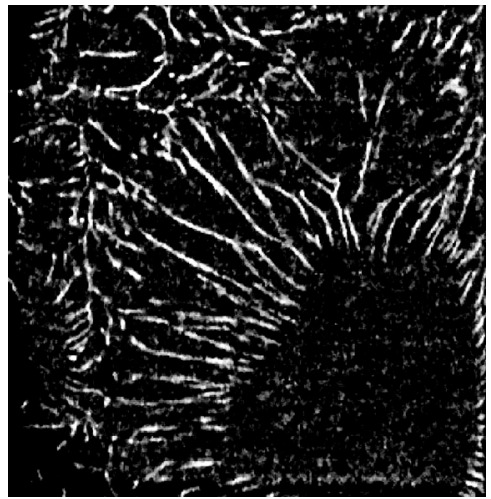

(b)

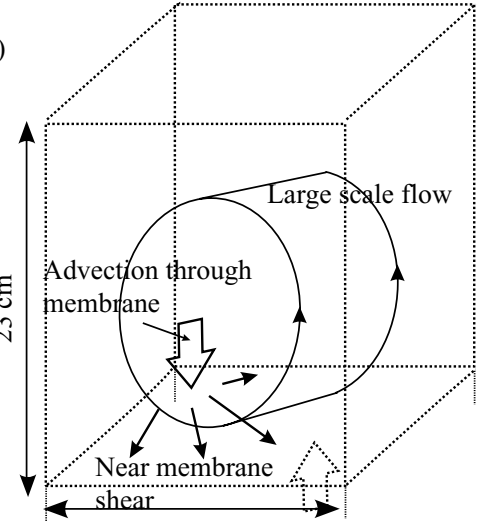

$(d)$

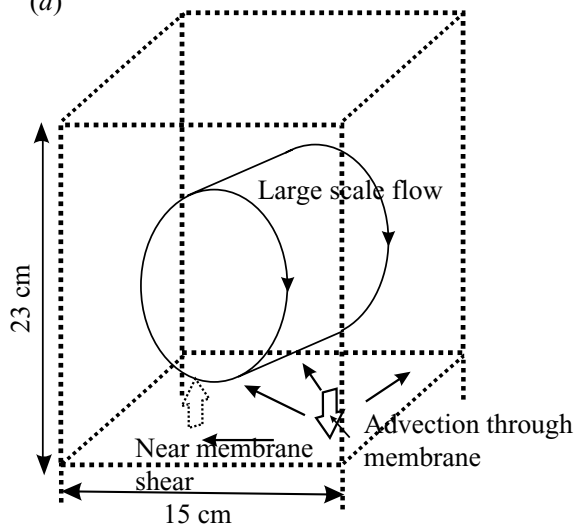

FIGURE 15. (a) The planform plume structure in the beginning of the combined regime in an experiment with $C_{T}^{0}=7 \mathrm{~g} \mathrm{I}^{-1}$. Diagonal orientation at $R a_{w}=2 \times 10^{11}$. (b) Schematic of the large-scale flow in $(a)$. (c) Planform plume structure in the combined regime at the beginning of an experiment with $C_{T}^{0}=3 \mathrm{gl}^{-1}$ at $R a_{w}=0.843 \times 10^{11}$. (d) Schematic of the large-scale flow in $(c)$. Parameters corresponding to the figures are shown in table 1 .

\section{Appendix A. The combined regime}

The combined regime occurs at driving potentials lower than those in the advection regime, but higher than those in the diffusion regime. This may be considered as a transition regime in which both diffusion and advection are important. In this regime, we sometimes observe plume-free regions indicating advection, though sometimes plumes cover the full membrane.

Figures 15(a) shows the plume structure at the beginning of the combined regime in one experiment with $C_{T}^{0}=7 \mathrm{~g}^{-1}$. At the $R a_{w}$ of these planforms, the corresponding $R a_{\delta}^{-1 / 3}$, both calculated with $\Delta C_{w}=\Delta C / 2$, is constant at around 0.14 (figure $7 b$ and table 1). Hence, if $\Delta C_{w}=\Delta C / 2$ in the experiment at the time of these planforms, the magnitude of flux and its scaling $\left((\Delta C / 2)^{4 / 3}\right)$ is the same as that in RBC. Note that in the case of experiments started with $C_{T}^{0}=10 \mathrm{gl}^{-1}$ too, the cubic scaling of flux in the advection regime changes to a $(\Delta C / 2)^{4 / 3}$ scaling, at similar driving potentials where the $C_{T}^{0}=7 \mathrm{~g}^{-1}$ experiments show $(\Delta C / 2)^{4 / 3}$ scaling (figure $7 b$ ). 
Figure 15(a) shows a plume-free circular patch in the top left-hand corner of the image, with radially aligned plumes around this patch. The plumes lose their alignment away from the patch. The near-membrane motion in the experiment could be seen to be along the alignment direction of the plumes. We infer these aligned plumes to be the signature of a large-scale flow impinging at the top left-hand corner. The largescale-flow schematic is shown in figure $15(b)$. The lower strength of the large-scale-flow (see table 1), due to the lower flux in this regime, than in the case of the advection regime results in a smaller plume, free area than that in the planforms of figure $8(a)$.

The planform plume structure after $30 \mathrm{~min}$ from the start of an experiment with initial top-tank concentration of $C_{T}^{0}=3 \mathrm{~g} \mathrm{l}^{-1}$ is shown in figure $15(c)$. If $\Delta C_{w}=(\Delta C-$ $\left.\Delta C_{m}\right) / 2$, for the value of $\Delta C_{w}=0.933 \mathrm{gl}^{-1}\left((\Delta \rho / \rho)_{w}=6.6 \times 10^{-4}\right)$ corresponding to the planform, figure $7(a)$ shows that $R a_{\delta}^{-1 / 3}$ is about 0.2 , larger than the value of 0.166 in the diffusion regime; the transport across the membrane at these driving potentials is not merely by pure diffusion. We hence classify this planform to be in the combined regime with $\Delta C_{w}=\Delta C / 2$ at $R a_{w}=0.843 \times 10^{11}$. The plumes are more widely spaced and are fewer in number than in figure $15(a)$. The lateral movement of plumes is slower compared to the $C_{T}^{0}=10 \mathrm{~g}^{-1}$ and $C_{T}^{0}=7 \mathrm{gl}^{-1}$ experiments. The presence of the plume-free patch shows that a large-scale flow impinges on the bottom right-hand side of the image; the schematic of the large-scale flow is shown in figure $15(d)$. The circular patch is seen to shift location in these experiments, indicating a changing large-scale-flow direction. This change of large-scale-flow orientation is in contrast to the observations in the advection regime where the stable orientation of the circulation was along the diagonal of the tank cross-section. Multiple circular patches are also seen sometimes during these experiments.

The phenomenology behind the combined regime is not completely clear from the present results. The planforms in figure 15(a) and 15(c) show plume-free regions where advection across the membrane is predominant and plume-covered regions where diffusion could be predominant. The presence of advection and diffusion across the membrane in the combined regime is also clear from the value of $R a_{\delta}^{-1 / 3}$ in figure $7(a)$. This dimensionless flux is larger than 0.166 in the combined regime, implying that the transport across the membrane is not purely by diffusion. At the same time, for the velocities in the mesh pore encountered in this regime, the numerical computations in Appendix D show a non-negligible concentration drop across the membrane, indicating appreciable diffusive transport. Hence, the transport of $\mathrm{NaCl}$ in this regime appears to be due to a combination of diffusion and advection. One possibility is that the combined regime is a mixed regime with advection and diffusion prominent in different parts of the membrane. If the area averaged profile is that in figure $5(b)$, then we obtain the dimensionless flux $R a_{\delta}^{-1 / 3} \simeq 0.14$, close to the RBC value. It is also possible that the rising plumes near the membrane cause a weak suction, creating a weak flow inside the membrane pores so that the diffusion drop in the membrane pore becomes negligible. The boundary layers on the membrane in such a situation will probably retain their no-through-flow character to give the $q \sim(\Delta C / 2)^{4 / 3}$ scaling. These inferences must be substantiated with further experiments.

\section{Appendix B. Integral relations for the boundary layers}

Consider a steady laminar indirect natural convection boundary layer with a normal advective flow at the wall (figure 14(b)). The boundary-layer equations are,

$$
\frac{\partial U}{\partial X}+\frac{\partial V}{\partial Y}=0
$$




$$
\begin{gathered}
U \frac{\partial U}{\partial X}+V \frac{\partial U}{\partial Y}=-\frac{1}{\rho} \frac{\partial P}{\partial X}+v \frac{\partial^{2} U}{\partial Y^{2}}, \\
\frac{1}{\rho} \frac{\partial P}{\partial Y}=g \beta\left(C_{T}-C\right), \\
U \frac{\partial C}{\partial X}+V \frac{\partial C}{\partial Y},=D \frac{\partial^{2} C}{\partial Y^{2}} .
\end{gathered}
$$

The boundary conditions, at the wall, $Y=0$, are

$$
U=0, V=V_{i}, C=C_{B},
$$

and at the edge of the velocity boundary layer, $Y=\delta_{v}$, are

$$
U=0, V=V_{\delta_{v}}, C=C_{T},
$$

where $V_{\delta_{v}}$ is the entrainment velocity at the edge of the velocity boundary layer and $P$ is the motion pressure. At the edge of the species boundary layer, $Y=\delta$,

$$
U=U_{\delta}, V=V_{\delta}, C=C_{T},
$$

where $V_{\delta}$ is the entrainment velocity at the edge of the species boundary layer.

Integrating (B 1) from the wall to $\delta_{v}(X)$ and applying the boundary conditions, we obtain the integral mass conservation equation as

$$
\frac{\mathrm{d}}{\mathrm{d} X} \int_{0}^{\delta_{v}(X)} U \mathrm{~d} Y+V_{\delta_{v}}-V_{i}=0 .
$$

Multiplying (B 1) by U, subtracting (B 2), and integrating the resulting equation from 0 to $\delta_{v}(X)$, we obtain the integral $X$-momentum equation as,

$$
\frac{\mathrm{d}}{\mathrm{d} X} \int_{0}^{\delta_{v}(X)} U^{2} \mathrm{~d} Y+\frac{1}{\rho} \int_{0}^{\delta_{v}(X)} \frac{\partial P}{\partial X} \mathrm{~d} Y+\left.v \frac{\partial U}{\partial Y}\right|_{Y=0}=0 .
$$

The equation expresses a balance of the horizontal momentum flux with the horizontal pressure gradient and the wall shear stress. Integrating (B4) from 0 to $\delta(X)$ and substituting the expression for $V_{\delta}$ from the integrated form of (B 1) up to $\delta$, the integral species equation is obtained as

$$
\frac{\mathrm{d}}{\mathrm{d} X} \int_{0}^{\delta(X)} U\left(C-C_{T}\right) \mathrm{d} Y+\left.D \frac{\partial C}{\partial Y}\right|_{Y=0}+V_{i} \Delta C=0,
$$

where $\Delta C=\Delta C_{w}=C_{T}-C_{B}$. Equation (B 10) expresses a balance between convection by the horizontal velocity, entrainment from the ambient, the diffusive flux and the advective flux.

Integrating the $Y$-momentum equation, (B 3), with respect to $Y$ and using the uniform concentration approximation along with $\left.P\right|_{Y=\delta}=0$, we obtain the motion pressure distribution in the species boundary layer as,

$$
P(X, Y)=-\rho g \beta \Delta C(\delta(X)-Y) .
$$

Equation (B 11) implies that under the uniform concentration approximation, the motion pressure at any horizontal location in the species boundary layer depends linearly on the vertical direction. 


\section{Appendix C. Uniform concentration approximation}

In high-Schmidt-number natural-convection boundary layers, the species boundarylayer thickness is much smaller than the velocity boundary-layer thickness. A weak advection will create a uniform wall concentration through the major portion of the species boundary layer (figure $14 C$ ). The thickness of the uniform wall concentration region, $\delta_{d b}$ can be estimated from mass balance as

$$
V_{i} X=\delta_{d b} U \text {. }
$$

The thickness of the concentration gradient region in the species boundary layer, $\delta_{d g}$ can be estimated by a balance of convection and diffusion as

$$
D \frac{\Delta C}{\delta_{d g}} X=U \delta_{d g} \Delta C .
$$

The ratio $\delta_{d b} / \delta_{d g}$ from (C1) and (C2) is

$$
\frac{\delta_{d b}}{\delta_{d g}}=V_{i} \sqrt{\frac{X}{U D}} .
$$

In dimensionless form,

$$
\frac{\tilde{\delta_{d b}}}{\tilde{\delta_{d g}}} \sim \tilde{V}_{i} \sqrt{\frac{\tilde{x}}{\tilde{u}}},
$$

where the non dimensionalization is done by the characteristic scales of laminar natural convection boundary layer with no wall normal advection, namely $L$, $\delta_{c} \sim L /\left(G r_{L} S c\right)^{1 / 5}, v_{c} \sim(D / L)\left(G r_{L} S c\right)^{1 / 5}, u_{c} \sim(D / L)\left(G r_{L} S c\right)^{2 / 5}$ (see (Rotem \& Classen 1969)). If $\tilde{x}$ and $\tilde{u}$ are of order one, $\tilde{\delta}_{d b} / \tilde{\delta}_{d g}$ is proportional to the parameter $\tilde{V}_{i}=V_{i} / v_{c}=P e_{i} /\left(G r_{L} S c\right)^{1 / 5}$, where $P e_{i}=V_{i} L / D$. Using (5.5), at the beginning of the advection-dominated regime, $V_{i}=0.0018 \mathrm{~cm} \mathrm{~s}^{-1}$ and $L=$ order of plume spacing $\sim 0.6 \mathrm{~cm}$, giving $\tilde{V}_{i} \sim 3$. This implies that $75 \%$ of the species boundary-layer thickness is of uniform concentration. For further analysis, we assume the species boundary layer to have a constant concentration $C_{B}$ throughout its thickness.

\section{Appendix D. Effect of advection on the concentration profile in the membrane}

The major assumption in the analysis of $\S 5.3$ is that there is no diffusive flux across the membrane at the velocities encountered in the mesh pores in the advection regime. This happens when the advection is strong enough to remove any concentration gradients in the mesh pores. The effect of an advection velocity on the concentration profile in a micro pore is studied in this section, to verify the absence of diffusive flux in the advection regime.

At any instant, the concentration distribution in a membrane pore with $C_{T}(t), C_{B}(t)$ and vertical velocity $V_{w}(t)$ as the boundary conditions at the ends of the pore will be given by the one-dimensional species equation. The solution shows an exponential dependence on the advection velocity as,

$$
\frac{C_{T}(t)-C(Y, t)}{C_{T}(t)-C_{B}(t)}=\frac{1-\exp \left(V_{w}(t)\left(Y-l_{m}\right) / D\right)}{1-\exp \left(-P e_{V_{w}}\right)}
$$

where, $P e_{V_{w}}=V_{w} l_{m} / D$ is the Péclet number based on the membrane thickness and $V_{w}=V_{i} / \Gamma$. The concentration distribution (D1) shows that, in the beginning of the advection regime when $V_{w} \sim 0.0072 \mathrm{~cm} \mathrm{~s}^{-1}$ and $P e_{V_{w}}=3.4,85 \%$ of the total 


$\begin{array}{clllrrr}V_{i}\left(\mathrm{~cm} \mathrm{~s}^{-1}\right) \times 10^{-3} & 0.5 & 1 & 2 & 3 & 4 & 5 \\ V_{w}=V_{i} / \Gamma\left(\mathrm{cm} \mathrm{s}^{-1}\right) \times 10^{-3} & 2 & 4 & 8 & 12 & 16 & 20 \\ P e_{V_{i}} & 0.238 & 0.4755 & 0.95 & 1.43 & 1.9 & 2.28\end{array}$

TABLE 2. Parameters for the computations to study the effects of advection on convection in a pore of the membrane. $V_{i}$ is the inflow boundary condition in the computation. $P e_{V_{i}}=V_{i} l_{m} / D$.
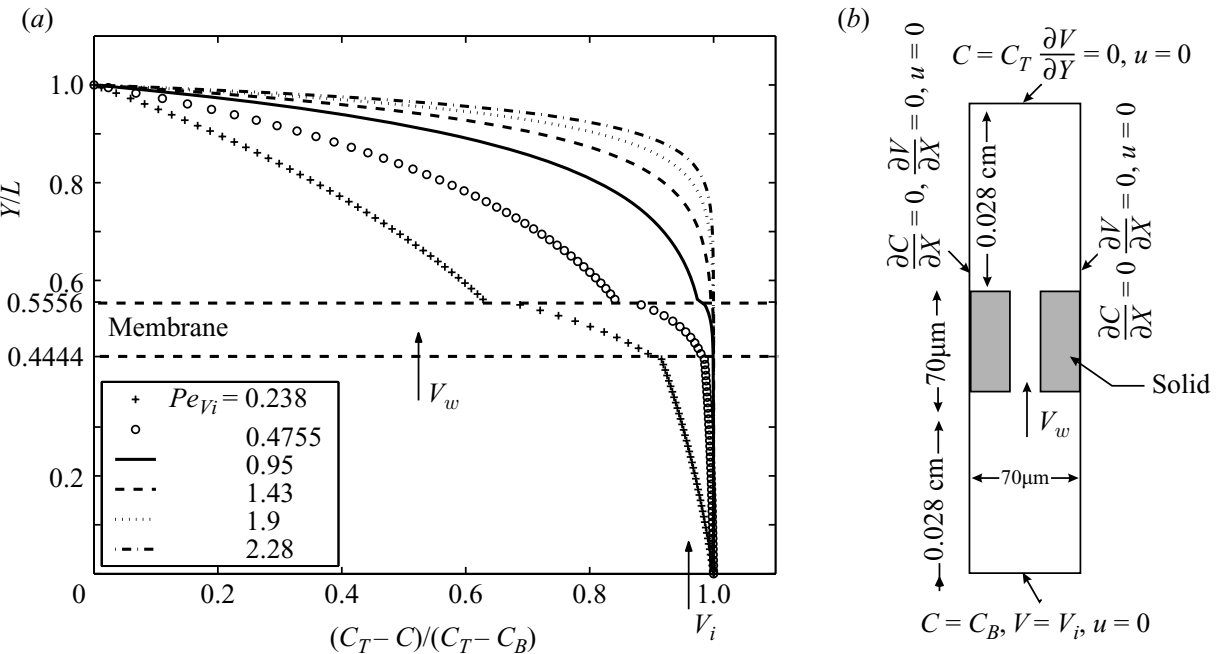

FIGURE 16. (a) Horizontally averaged concentration profiles across the membrane obtained from computations at different advection velocities. (b) Geometry and boundary conditions for the computations.

concentration drop occurs by half the pore height. As $\Delta C$ occurs across the membrane and boundary-layer combination in experiments, the membrane pore will not be subject to the total $\Delta C$; the above estimate is an overestimate.

To find more realistic estimates, two-dimensional computations were conducted on a slit having the open area factor $\Gamma=0.25$ (the same as that of the $140 \mathrm{~s}$ membrane). The domain of computation and boundary conditions, corresponding to a unit cell of the membrane, are shown in figure $16(b)$. The computational domain height above and below the membrane was taken to be of the same order as the estimated boundary-layer thickness in the experiments. Buoyancy effects were neglected. The inflow velocity boundary condition in the computations, chosen to cover the range of advection velocities $V_{i}$ in experiments, is shown in table 2 . The velocities in the advection regime in experiments are $0.76 \times 10^{-3} \mathrm{~cm} \mathrm{~s}^{-1} \leqslant V_{i} \leqslant 1.8 \times 10^{-3} \mathrm{~cm} \mathrm{~s}^{-1}$, i.e. $0.36 \leqslant P e_{V_{i}} \leqslant 0.83$, where $P e_{V_{i}}=V_{i} l_{m} / D$.

Figure 16 shows the horizontally averaged concentration profiles from the computations, for the range of velocities studied. Negligible drop in concentration $(\sim 2 \%)$ occurs across the membrane at $P e_{V_{i}}=0.829$, corresponding to the beginning of the advection regime. Notice the absence of concentration drop below the membrane at this advection velocity. $P e_{V_{i}} \sim 0.353$ corresponds to the end of the advection regime, when the cubic scaling of flux starts changing to a $4 / 3$ scaling. At this $P e_{V_{i}}$, the concentration drop across the membrane is less than a quarter of the total concentration drop. This too is an overestimate as we have neglected the increase 
in the boundary-layer thickness, as lower $P e_{V_{i}}$ occurs at lower $R a_{w}$. Therefore, for the through-flow velocities in the cubic scaling regime of the experiments, the contribution of diffusive flux across the mesh is negligible at the beginning of the experiment, increases with reducing advection velocities, but the concentration drop across the membrane will always be less than $25 \%$ of the total concentration drop (see Puthenveettil 2004 for details). We conclude that the assumption of negligible concentration drop across the membrane, above the membrane in the region of down flow and below the membrane in the region of up flow (as in figure $3 b$ ), is a valid approximation in the advection regime.

\section{REFERENCES}

Adrian, R. J., Ferreira, R. T. D. S. \& Boberg, T. 1986 Turbulent thermal convection in wide horizontal fluid layers. Exps. Fluids 4, 121-141.

AfZal, N. \& Hussain, T. 1986 Effects of large suction in natural convection boundary layer on a horizontal layer. Warme Stoffubertragung 20, 203-206.

Bizon, C., Werne, J., Predtechensky, A., Julien, K., McCormick, W., Swift, J. \& Swinney, H. 1997 Plume dynamics in quasi-2d turbulent convection. Chaos 7, 107-124.

Buch, K. A. \& Dahm, W. J. A. 1996 Experimental study of the fine scale structure of conserved scalar mixing in turbulent shear flows. Part 1. Sc 》1. J. Fluid Mech. 317, 21-71.

Chavanne, X., Chilla, F., Chabaud, B., Castaing, B. \& Hebral, B. 2001 Turbulent RayleighBénard convection in gaseous and liquid He. Phys. Fluids 13 (5), 1300-1320.

Clarke, J. F. \& Riley, N. 1975 Natural convection induced in a gas by the presence of a hot porous horizontal surface. Q.J. Mech. Appl. Maths 28 (4), 375-396.

CRImaldi, J. P. 1997 The effect of photobleaching and velocity fluctuations on single-point LIF measurements. Exps. Fluids 23, 325-330.

Dahm, W. J. A. \& Dimotakis, P. E. 1987 Measurements of entrainment and mixing in turbulent jets. AIAA J. 25, 1216-1223.

DeARDorfF, J. W. 1970 Convective velocity and temperature scales for the unstable planetary boundary layer and for Rayleigh convection. J. Atmos. Sci. 27, 1211-1213.

Du, Y. \& Tong, P. 2000 Turbulent thermal convection in a cell with ordered rough boundaries. J. Fluid Mech. 407, 57-84.

Frank, H. \& Althoen, S. C. 2002 Statistics, Concepts and Applications. Cambridge University Press.

Funfschilling, D. \& Ahlers, G. 2004 Plume motion and large scale circulation in a cylindrical Rayleigh-Bénard cell. Phys. Rev. Lett. 92, 194502.

Gill, W. N., Zeh, D. W. \& Casal, E. D. 1965 Free convection on a horizontal plate. Z. Angew. Math. Phys. 16, 539-541.

Goldstein, R. J., Chiang, H. D. \& See, D. L. 1990 High-Rayleigh-number convection in a horizontal enclosure. J. Fluid Mech. 213, 111-126.

Husar, R. B. \& Sparrow, E. M. 1968 Patterns of free convection flow adjacent to horizontal heated surfaces. Intl J. Heat Mass Transfer 11, 1208-1211.

Kadanoff, L. P. 2001 Turbulent heat flow: structures and scaling. Phys. Today 54(8), 34-39.

KerR, R. M. 1996 Rayleigh number scaling in numerical convection. J. Fluid Mech. 310, 139-179.

LIDE, D. R. (ed.) 2001 CRC Handbook of Chemistry and Physics, 80th edn. CRC Press.

NiELD, D. A. 1987 Througflow effects in the Rayleigh-Bénard convective instbiltiy problem. J. Fluid Mech. 185, 353-360.

Niemela, J. J. \& Sreenivasan, K. R. 2003 Confined turbulent convection. J. Fluid Mech. 481, $355-384$

Niemela, J. J. \& SReenivasan, K. R. 2006 Turbulent convection at high Rayleigh numbers and aspect ratio 4. J. Fluid Mech. 557, 411-422.

Niemela, J. J., Skrbek, L., Sreenivasan, K. R. \& Donelly, R. J. 2000 Turbulent convection at very high Rayleigh numbers. Nature 404, 837-840.

Niemela, J. J., Skrbek, L., Sreenivasan, K. R. \& Donnely, R. J. 2001 The wind in confined thermal convection. J. Fluid Mech. 449, 169-178.

ORION 1999 Sensorlink ${ }^{T M}$ Model PCM100 Instruction Manual. ORION Research. 
PuthenveEtTiL, B. A. 2004 Investigations on high Rayleigh number turbulent free-convetion. PhD thesis, Indian Institute of Science, Bangalore, http://etd.ncsi.iisc.ernet.in/handle/2005/140.

Puthenveettil, B. A. \& Arakeri, J. H. 2005 Plume structure in high-Rayleigh-number convection. J. Fluid Mech. 542, 217-249.

Puthenveettil, B. A., Ananthakrishna, G. \& Arakeri, J. H. 2005 The multifractal nature of plume structure in high-Rayleigh-number convection. J. Fluid Mech. 526, 245-256.

Rotem, Z. \& Classen, L. 1969 Natural convection above unconfined horizontal surfaces. J. Fluid Mech. 39, 173-192.

ShVARTSBlat, D. L. 1969 Steady convective motions in a plane horizontal fluid layer with permeable boundaries. Izv. Akad. Nauk SSSR Mekh. Zhid. i Gaza 4 (5), 84-90.

Siggia, E. D. 1994 High Rayleigh number convection. Annu. Rev. Fluid Mech. 26, 137-168.

Spangenberg, W. G. \& Rowland, W. G. 1961 Convective circulation in water induced by evaporative cooling. Phys. Fluids 4 (6), 743-750.

SPARrow, E. \& HuSAR, R. 1969 Longitudinal vortices in natural convection flow on inclined plates. J. Fluid Mech. 37, 251-255.

Stringano, G., Pascazio, G. \& Verzicco, R. 2006 Turbulent thermal convection over grooved plates. J. Fluid Mech. 557, 307-336.

Sun, C., Ren, L.-Y., Song, H. \& XIA, K.-Q. 2005 Heat transport by turbulent Rayleigh-Bénard convection in $1 \mathrm{~m}$ diameter cylindrical cells of widely varying aspect ratio. J. Fluid Mech. $\mathbf{5 4 2}$, $165-174$.

Theerthan, S. A. \& Arakeri, J. H. 1998 A model for near-wall dynamics in turbulent RayleighBénard convection. J. Fluid Mech. 373, 221-254.

Theerthan, S. A. \& Arakeri, J. H. 2000 Plan form structure and heat transfer in turbulent free convection over horizontal surfaces. Phys. Fluids 12, 884-894.

Townsend, A. 1959 Temperature fluctuations over a heated horizontal surface. J. Fluid Mech. 5, 209-211.

XIA, K. Q., Lam, S. \& Zhou, S. Q. 2002 Heat flux measurement in high Prandtl number turbulent Rayleigh-Bénard convection. Phys. Rev. Lett. 88, 064501.

XIA, K. Q., Sun, C. \& ZHou, S. Q. 2003 Particle image velocimetry measurement of the velocity field in turbulent thermal convection. Phys. Rev. E 68, 066303.

Young, Y.-N., Tufo, H., Dubey, A. \& Rosner, R. 2001 On the miscible Rayleigh-Taylor instability: two- and three-dimensions. J. Fluid Mech. 447, 377-408.

Zhou, Q., Sun, C. \& XIA, K. Q. 2007 Morphological evolution of thermal plumes in turbulent Rayleigh-Bénard convection. Phys. Rev. Lett. 98, 074501.

Zocchi, G., Moses, E. \& Libchaber, A. 1990 Coherent structures in turbulent convection, an experimental study. Physica A 166, 387-407. 